TRANSACTIONS OF THE

AMERICAN MATHEMATICAL SOCIETY

Volume 354, Number 4, Pages 1511-1548

S 0002-9947(01)02909-9

Article electronically published on November 20, 2001

\title{
TRACTOR CALCULI FOR PARABOLIC GEOMETRIES
}

\author{
ANDREAS ČAP AND A. ROD GOVER
}

\begin{abstract}
Parabolic geometries may be considered as curved analogues of the homogeneous spaces $G / P$ where $G$ is a semisimple Lie group and $P \subset G$ a parabolic subgroup. Conformal geometries and CR geometries are examples of such structures. We present a uniform description of a calculus, called tractor calculus, based on natural bundles with canonical linear connections for all parabolic geometries. It is shown that from these bundles and connections one can recover the Cartan bundle and the Cartan connection. In particular we characterize the normal Cartan connection from this induced bundle/connection perspective. We construct explicitly a family of fundamental first order differential operators, which are analogous to a covariant derivative, iterable and defined on all natural vector bundles on parabolic geometries. For an important subclass of parabolic geometries we explicitly and directly construct the tractor bundles, their canonical linear connections and the machinery for explicitly calculating via the tractor calculus.
\end{abstract}

\section{INTRODUCTION}

Parabolic geometries form a large class of geometric structures which include, for example, projective, conformal, almost Grassmannian, almost quaternionic, and codimension one $\mathrm{CR}$-structures. A unifying feature of these structures is that they admit a principal bundle with structure group a parabolic subgroup $P$ of a semisimple Lie group $G$ and a Cartan connection on that principal bundle. Both the bundle and connection are uniquely determined by a normalization condition on the curvature of the Cartan connection. The construction of these normal Cartan connections has a rather long history, going back to E. Cartan for projective, conformal and three dimensional CR-structures.

The construction of canonical Cartan connections for all parabolic geometries is obtained (under some minor restrictions) in the work of N. Tanaka [31. The result in full generality has been obtained by T. Morimoto 27. from a general study of geometric structures on filtered manifolds, and independently in [6].

While constructing a normal Cartan connection solves the equivalence problem (at least in principle), it is only the first step towards the understanding of a geometric structure. Indeed, it is rather difficult to use a Cartan connection to deal with many natural geometric problems, such as, for example, a description of polynomial invariants of a structure, or the related problem of constructing differential operators which are intrinsic to the structure (invariant operators). Recent interest

Received by the editors July 17, 2000.

2000 Mathematics Subject Classification. Primary 53B15, 53C05, 53C07, 53C15; Secondary 32V05, 53A20, 53A30, 53A40, 53A55.

Key words and phrases. Parabolic geometry, Cartan connection, tractor bundle, tractor calculus, invariant differential operator, invariant calculus. 
in the construction of polynomial invariants of parabolic geometries was initiated by C. Fefferman [14], who wanted to access CR invariants for the asymptotic expansion of the Bergman kernel on a domain in $\mathbb{C}^{n}$. Although the asymptotic expansion of the Bergman kernel is now essentially understood [24] the CR invariants problem itself is still open.

Systematic ways to use Cartan connections in a relatively direct manner, to attack geometrical problems as stated above, have been developed only recently, see e.g. [8]. This has led to remarkable general results on invariant differential operators, see [9]. However for these developments substantial new ingredients (such as semiholonomic jet-modules) were also required. Earlier attempts to use the similarity of different parabolic geometries to study various structures in a uniform way were made for example in 28, [16], and 44 for the subclass of irreducible parabolic geometries (usually called almost Hermitian symmetric or AHS-structures).

There is another basic approach to parabolic geometries. This approach usually is known by the name "tractor calculus" or "local twistor calculus". This approach is based on the fact that certain natural vector bundles associated to a parabolic geometry admit canonical linear connections. For conformal and projective structures this was already noticed by Cartan, see [10, and it is a central ingredient in the work of T. Thomas [32]. For projective and conformal structures, Thomas used bundles of that type to construct some special invariant operators (now called tractor- $D$ operators) which are very powerful, for example because they can be iterated. Although these operators involve the canonical linear connection (which is equivalent to the Cartan connection) they are not simply a consequence of the connection but involve additional ingredients. The so-called "local twistor connection" (see [11]) is another connection along these lines which was also found independently of either source and forms part of the twistor theory machinery.

In [2] Thomas' calculus for conformal and projective structures was rediscovered and put into a more modern setting. This calculus was further developed in [18] and used there to produce an almost complete solution to the invariants problem for even dimensional conformal structures. The corresponding invariants problem for projective structures was completely solved by tractor calculus [17. Tractor calculus was later extended to CR structures [22, 19] and applied to the construction of CR invariant powers of the sub-Laplacian. It should be remarked that for these problems there is the alternative approach of the Fefferman-Graham ambient metric constructions, which leads to a complete description of polynomial invariants for odd-dimensional conformal structures, see 3. However, for even-dimensional conformal and $\mathrm{CR}$-structures the ambient metric construction is obstructed at finite order. Tractor calculus can be used to proliferate invariants "beyond the obstruction" to the ambient metric construction. Work is in progress with Hirachi 20] to use tools from tractor calculus to solve such CR invariants problems.

Tractor calculi for quaternionic, almost Grassmannian and related structures were touched on in [1 2] and then developed more fully in [21. In the latter article the calculus was used to explicitly construct a new family of invariant differential operators. Tractor and twistor calculus plays a key role in the so-called "curved translation principle" [13, 12, which is a scheme for producing very large classes of invariant differential operators. On the other hand in [5] tractor calculus for conformal hypersurfaces and elliptic theory has enabled the construction of conformally invariant pseudo-differential operators. 
While covering many important examples, the structures for which versions of tractor calculus have been developed up to now are only a small proportion of the wide variety of parabolic geometries. Moreover, all known versions worked for one concrete structure only, and all developments up to now were on a case-by-case basis, just using the visible similarities between various structures. In brief the results of this article are as follows: We develop a (normal) tractor calculus for all parabolic geometries and do this in a uniform manner. We provide a practical means of testing whether a candidate for a tractor calculus is the normal calculus. As part of the calculus we produce a new first order invariant operator, show how to calculate with this and describe an immediate application. Finally we give, for a large class of structures, a direct construction of the tractor calculus (i.e. a construction that avoids first constructing the Cartan bundle and connection). We conclude this introduction by elaborating on these points.

We start by introducing a uniform setting for tractor calculus for all parabolic geometries. The main ingredient for this is the so-called adjoint tractor bundle, which exists for any type of parabolic geometry and provides the basis for our calculus. After introducing these bundles and the appropriate linear connections (called tractor connections) on them, we show that we may recover from them a principal $P$-bundle endowed with a Cartan connection. Next, we characterize the usual normalization condition on Cartan connections in terms of the curvature of the tractor connection, thus arriving at a characterization of the normal adjoint tractor bundle, which needs no further reference to the Cartan bundle. This is important since in many cases it is rather easy to construct the tractor bundles and connections directly.

The key to the applications in [18] was not Thomas' (second order) tractor- $D$ operator but a new first order operator. In [19] this was referred to as the "double$D$ " operator and some analogous operators were also constructed for CR structures. In section 3, we vastly generalize these operators and develop an invariant calculus based on tractor bundles and in particular on the adjoint tractor bundle. More precisely, for any vector bundle $\mathcal{W}$ associated to the Cartan bundle, we introduce a first order differential operator called the fundamental $D$-operator, which maps smooth sections of the bundle to smooth sections of $\mathcal{A}^{*} \otimes \mathcal{W}$, where $\mathcal{A}$ denotes the adjoint tractor bundle. These fundamental $D$-operators have many features in common with covariant derivatives on vector bundles associated to a principal bundle endowed with a principal connection. In particular, they can be iterated and they behave naturally with respect to all constructions with associated vector bundles. The moral of this is that once one is willing to replace the tangent bundle by the adjoint tractor bundle (which has the tangent bundle as a quotient), then one is in a situation closely analogous to the case of a principal connection. It should be pointed out that, in contrast, the tractor connection only gives a covariant derivative on bundles $\mathcal{V}$ associated to $G$-modules and takes values in $T^{*} M \otimes \mathcal{V}$ and so cannot be iterated. As an application, we prove that knowing the fundamental $D$-operator on a bundle $\mathcal{W}$ and the tractor connection on the adjoint tractor bundle is sufficient to record the infinite jet of a section of $\mathcal{W}$ in an invariant way as a section of a fairly manageable bundle. Moreover, if $\mathcal{W}$ is a subquotient of a tensor bundle or in the case of the infinite jet of the structure itself (which is relevant for finding invariants) knowledge of the tractor connection alone is sufficient. This is a fundamental step towards the invariants problem for parabolic geometries. 
Finally, in section 4 we give a direct and uniform construction of adjoint tractor bundles for all irreducible parabolic geometries. Checking the normality of these is an application of the results of section 2 This is the first direct construction of the tractor bundles and their connections which works for an entire class of structures. This is presented in a notation based around the equivalence class of preferred connections on the tangent bundle of the underlying geometry and so enabling results to be interpreted directly in terms of tensors and these standard tools of differential geometry. In particular the fundamental $D$-operator is described explicitly in this language.

\section{TRACtor BUndLes AND tRaCtor CONNECTIONS}

2.1. The basic input needed to specify a parabolic geometry is a semisimple Lie group $G$ together with a so-called $|k|$-grading of the Lie algebra $\mathfrak{g}$ of $G$, that is,

$$
\mathfrak{g}=\mathfrak{g}_{-k} \oplus \cdots \oplus \mathfrak{g}_{0} \oplus \cdots \oplus \mathfrak{g}_{k},
$$

which has the properties that no simple ideal of $\mathfrak{g}$ is contained in $\mathfrak{g}_{0}$ and the subalgebra $\mathfrak{p}_{+}=\mathfrak{g}_{1} \oplus \cdots \oplus \mathfrak{g}_{k}$ is generated as a Lie algebra by $\mathfrak{g}_{1}$. We will also need the decreasing filtration corresponding to this grading, which has the form $\mathfrak{g}=\mathfrak{g}^{-k} \supset \cdots \supset \mathfrak{g}^{k}$, where $\mathfrak{g}^{i}=\mathfrak{g}_{i} \oplus \cdots \oplus \mathfrak{g}_{k}$. Then we define $G_{0} \leq P$ to be the subgroups of $G$ consisting of all elements whose adjoint action preserves the grading, respectively the filtration, of $\mathfrak{g}$, i.e.

$$
\begin{aligned}
G_{0} & =\left\{g \in G: \operatorname{Ad}(g)\left(\mathfrak{g}_{i}\right) \subset \mathfrak{g}_{i} \quad \forall i=-k, \ldots, k\right\}, \\
P & =\left\{g \in G: \operatorname{Ad}(g)\left(\mathfrak{g}^{i}\right) \subset \mathfrak{g}^{i} \quad \forall i=-k, \ldots, k\right\} .
\end{aligned}
$$

It follows immediately that $G_{0}$ corresponds to the Lie subalgebra $\mathfrak{g}_{0} \leq \mathfrak{g}$, while $P$ corresponds to the Lie subalgebra $\mathfrak{p}=\mathfrak{g}^{0} \leq \mathfrak{g}$. Moreover, it turns out that $P$ is the semidirect product of $G_{0}$ with a vector group, so $P$ and $G_{0}$ are homotopy equivalent. More precisely, one proves (see [6, proposition 2.10]) that for any element $g \in P$ there exist unique elements $g_{0} \in G_{0}$ and $Z_{i} \in \mathfrak{g}_{i}$ for $i=1, \ldots, k$ such that $g=$ $g_{0} \exp \left(Z_{1}\right) \ldots \exp \left(Z_{k}\right)$.

A parabolic geometry, corresponding to $G$ and the grading of $\mathfrak{g}$, on a smooth manifold $M$ is given by a principal $P$-bundle $\mathcal{G} \rightarrow M$ together with a Cartan connection $\omega \in \Omega^{1}(\mathcal{G}, \mathfrak{g})$ of type $(G, P)$ on $\mathcal{G}$. That is, $\omega$ is a $\mathfrak{g}$-valued one-form on $\mathcal{G}$ such that:

(1) $\omega\left(\zeta_{A}\right)=A$, where $\zeta_{A}$ is the fundamental vector field corresponding to $A \in \mathfrak{p}$,

(2) $\left(r^{g}\right)^{*} \omega=\operatorname{Ad}\left(g^{-1}\right) \circ \omega$, where $r^{g}$ denotes the principal right action of $g \in P$,

(3) $\left.\omega\right|_{T_{u} \mathcal{G}} T_{u} \mathcal{G} \rightarrow \mathfrak{g}$ is a linear isormorphism for all $u \in \mathcal{G}$.

Moreover, the curvature of $\omega$ has to satisfy a normalization condition which we will discuss in section 2.10

In fact, there are various "natural" choices for the group $G$ once the Lie algebra $\mathfrak{g}$ is given. Among them is the (unique) simply connected and connected group, the group $\operatorname{Aut}(\mathfrak{g})$ of all Lie algebra automorphisms of $\mathfrak{g}$ and the adjoint group $\operatorname{Int}(\mathfrak{g})$ of inner automorphisms of $\mathfrak{g}$, which is just the connected component of the identity of $\operatorname{Aut}(\mathfrak{g})$. Changing between various groups with the same Lie algebra does not change the corresponding structure a lot. Usually it amounts to preserving an additional orientation or a structure similar to a spin structure, etc. We will comment more on this problem in 2.3 below. It should also be pointed out that locally no problems arise from any particular choice of group. Over a contractible 
open subset of a manifold one can always switch from one group to another (possibly by making choices).

Usually, principal bundles equipped with Cartan connections over smooth manifolds are not easy to interpret geometrically, and proving the existence of a canonical Cartan connection for a given geometric structure is often highly non-trivial. In the case of parabolic geometries, this problem has been completely solved, for example in [6. The subject of that paper is to construct a parabolic geometry on a smooth manifold $M$ from an underlying structure, which can be described as follows: First one needs a filtration $T M=T^{-k} M \supset T^{-k+1} M \supset \cdots \supset T^{-1} M$ such that the rank of $T^{i} M$ equals the dimension of $\mathfrak{g}_{i} \oplus \cdots \oplus \mathfrak{g}_{-1}$ and such that the Lie bracket of a section of $T^{i} M$ with a section of $T^{j} M$ is a section of $T^{i+j} M$. This Lie bracket of vector fields on $M$ induces an algebraic Lie bracket on the associated graded vector bundle $\operatorname{gr}(T M)=T^{-k} M / T^{-k+1} M \oplus \cdots \oplus T^{-2} M / T^{-1} M \oplus T^{-1} M$. One then has to put on that exactly the additional structure which characterizes $\mathfrak{g}_{-}=\mathfrak{g}_{-k} \oplus \cdots \oplus \mathfrak{g}_{-1}$ as a $G_{0}$-module. Except for two structures (projective structures and a contact-analog of those) for which one has to make an additional choice to fix the structure, this is the complete underlying structure.

Here we construct a parabolic geometry on a manifold starting from a different set of underlying data. The underlying data we use here consists of certain vector bundles (the so-called tractor bundles) over a manifold endowed with an appropriate linear connection (a tractor connection). We show that these tractor bundles are the induced bundles of the canonical principal bundle which correspond to representations of the group $G$, viewed as representations of the subgroup $P$. Among these bundles a central role is played by the adjoint tractor bundle, which corresponds to the adjoint representation.

2.2. Definition. (1) Let $M$ be a smooth manifold of the same dimension as $\mathfrak{g} / \mathfrak{p}$. An adjoint tractor bundle over $M$ is a smooth vector bundle $\mathcal{A} \rightarrow M$, which is endowed with a decreasing filtration $\mathcal{A}=\mathcal{A}^{-k} \supset \mathcal{A}^{-k+1} \supset \cdots \supset \mathcal{A}^{k}$ by smooth subbundles and an algebraic Lie bracket $\{\}:, \mathcal{A} \otimes \mathcal{A} \rightarrow \mathcal{A}$, such that $\mathcal{A}$ is a locally trivial bundle of filtered Lie algebras modeled on $\mathfrak{g}$. This means that we have local trivializations $\left.\mathcal{A}\right|_{U} \rightarrow U \times \mathfrak{g}$ for $\mathcal{A}$ which are compatible with the filtration and the bracket.

(2) Let $\mathcal{A} \rightarrow M$ be an adjoint tractor bundle over $M$, and let $G$ be a group with Lie algebra $\mathfrak{g}$ with the subgroups $G_{0} \leq P \leq G$ as in 2.1 above. An adapted frame bundle for $\mathcal{A}$ corresponding to $G$ is a smooth principal bundle $\mathcal{G} \rightarrow M$ with structure group $P$ such that $\mathcal{A}=\mathcal{G} \times{ }_{P} \mathfrak{g}$, the associated bundle with respect to the adjoint representation of $P$ on $\mathfrak{g}$.

Proposition 2.3. If $\mathcal{A} \rightarrow M$ is an adjoint tractor bundle, then there exists a canonical adapted frame bundle $\mathcal{G} \rightarrow M$ for $\mathcal{A}$ corresponding to the group $G=$ Aut $(\mathfrak{g})$ of all Lie algebra automorphisms of $\mathfrak{g}$.

Proof. Constructing subgroups $G_{0} \leq P \leq G$ as in 2.1 above, gives $G_{0}$ as the group of all automorphisms of the graded Lie algebra $\mathfrak{g}$ and $P$ as the group of all automorphisms of the filtered Lie algebra $\mathfrak{g}$. Now let $\mathcal{G}$ be the set of all isomorphisms of filtered Lie algebras from $\mathfrak{g}$ to some fiber of $\mathcal{A}$. Obviously, this can be viewed as a subbundle of the linear frame bundle of $\mathcal{A}$, which consists of all linear isomorphisms (of vector spaces) from $\mathfrak{g}$ to some fiber of $\mathcal{A}$. In particular, this implies that the 
obvious projection $p: \mathcal{G} \rightarrow M$ is a smooth map. Moreover, by our assumptions on $\mathcal{A}$, this projection has smooth local sections, so it is a surjective submersion.

Next, we get a canonical right action of $P$ on $\mathcal{G}$ by composition from the right. Since $P$ is the group of all automorphisms of the filtered Lie algebra $\mathfrak{g}$ this action is free and transitive on each fiber, so $p: \mathcal{G} \rightarrow M$ is a smooth principal $P$-bundle.

A point $u \in \mathcal{G}$ with $p(u)=x \in M$ by definition is an isomorphism $\mathfrak{g} \rightarrow \mathcal{A}_{x}$ of filtered Lie algebras. Thus we get a canonical smooth map $\mathcal{G} \times \mathfrak{g} \rightarrow \mathcal{A}$ by mapping $(u, A)$ to $u(A)$. If $\varphi \in P$ is an automorphism of $\mathfrak{g}$, then $\left(u \circ \varphi, \varphi^{-1}(A)\right)$ is also mapped to $u(A)$, so this induces a smooth vector bundle homomorphism $\mathcal{G} \times_{P} \mathfrak{g} \rightarrow \mathcal{A}$, which covers the identity on $M$ and is a linear isomorphism in each fiber. Thus $\mathcal{A}$ is the associated bundle to $\mathcal{G}$ with respect to the canonical action of $P$ on $\mathfrak{g}$ which coincides with the adjoint action.

Remark. As we mentioned in section 2.1, for particular applications it may be convenient or necessary to work with a group $G$ (with Lie algebra $\mathfrak{g}$ ) which is different from $\operatorname{Aut}(\mathfrak{g})$. For all such groups a similar construction of adapted frame bundles as in the proposition above is possible: Let us first consider the case of subgroups of $\operatorname{Aut}(\mathfrak{g})$ which still have Lie algebra $\mathfrak{g}$, i.e. which contain the connected component $\operatorname{Int}(\mathfrak{g})$ of the identity in $\operatorname{Aut}(\mathfrak{g})$. If $G$ is such a group, then one just has to characterize $G$ as a group of automorphisms of $\mathfrak{g}$, which usually amounts to preserving an orientation of $\mathfrak{g}$, or some similar structure. Then one has to equip $\mathcal{A}$ with the analog of that structure and in the construction of the above proposition restrict to those isomorphisms $\mathfrak{g} \rightarrow \mathcal{A}_{x}$ which are compatible with that structure. This procedure is needed for example in passing from conformal manifolds to oriented conformal manifolds.

Now if $G$ is a general Lie group with Lie algebra $\mathfrak{g}$, then the adjoint action defines a homomorphism Ad $: G \rightarrow \operatorname{Aut}(\mathfrak{g})$. Let $\underline{G}=\operatorname{Ad}(G) \subset \operatorname{Aut}(\mathfrak{g})$ be the image of $G$ under this homomorphism, and let $H \subset G$ be the kernel of Ad. By construction, we then get surjective homomorphisms $P \rightarrow \underline{P}$ and $G_{0} \rightarrow \underline{G}_{0}$ which both have kernel $H$. As described above, we get an adapted frame bundle $\underline{\mathcal{G}} \rightarrow M$ with group $\underline{P}$. Using this, we can construct a principal $\underline{G}_{0}$-bundle $\underline{\mathcal{G}}_{0} \rightarrow M$ as the space $\underline{\mathcal{G}} / \underline{P}_{+}$of orbits of the group $\underline{P}_{+}$, the image of $\exp \left(\mathfrak{p}_{+}\right)$in $\underline{P}$, and the canonical projection $\underline{\mathcal{G}} \rightarrow \underline{\mathcal{G}}_{0}$ is a principal $\underline{P}_{+}$-bundle. Now one has to choose an additional structure, namely an extension of the principal bundle $\underline{\mathcal{G}}_{0}$ to the group $G_{0}$, i.e. a principal $G_{0}$-bundle $\mathcal{G}_{0} \rightarrow M$ together with a homomorphism $\mathcal{G}_{0} \rightarrow \underline{\mathcal{G}}_{0}$ over the projection $G_{0} \rightarrow \underline{G}_{0}$. This corresponds to choosing a structure similar to a spin structure in Riemannian geometry. Having made this choice, we then get the adapted frame bundle $\mathcal{G}$ with group $P$ as the pullback of $\underline{\mathcal{G}} \rightarrow \underline{\mathcal{G}}_{0}$ along the map $\mathcal{G}_{0} \rightarrow \underline{\mathcal{G}}_{0}$. For example, this is exactly the process needed to pass from oriented conformal manifolds to oriented conformal spin-manifolds.

These comments are broad since the details of the possible structures depend on the concrete choices of $\mathfrak{g}$ and so it is not possible to treat all cases in a uniform way. For our present purposes, the most important point is that there is clearly no problem at a local level. Furthermore, these details will have no bearing on our general treatment below so we henceforth simply assume that for a specific structure we have fixed a suitable group $G$ and that the necessary accompanying structure has been incorporated into the definition of an adjoint tractor bundle $\mathcal{A} \rightarrow M$. Thus, we have always adapted frame bundles which correspond to the group $G$. 
2.4. Definition. Let $\mathcal{A} \rightarrow M$ be an adjoint tractor bundle over $M$ and let $\mathcal{G} \rightarrow M$ be an adapted frame bundle for $\mathcal{A}$ corresponding to a group $G$ with Lie algebra $\mathfrak{g}$, and consider the subgroup $P \leq G$ as before. Let $\mathbb{V}$ be a finite dimensional effective $(\mathfrak{g}, P)$-module, i.e. a $P$-module such that the infinitesimal action of $\mathfrak{p}$ on $\mathbb{V}$ extends to an effective action of $\mathfrak{g}$. We define the $\mathbb{V}$-tractor bundle $\mathcal{T}=\mathcal{T}_{\mathbb{V}}$ for $\mathcal{A}$ to be the associated bundle $\mathcal{G} \times{ }_{P} \mathbb{V}$.

Note that any infinitesimally effective representation of the group $G$ is effective as a $(\mathfrak{g}, P)$-module and in the case where $\mathfrak{g}$ is simple, effectivity just means nontriviality.

Let $\mathcal{T}$ be the $\mathbb{V}$-tractor bundle for $\mathcal{A}$. The $\mathfrak{g}$-action on $\mathbb{V}$ which is compatible with the $P$-action then gives rise to a vector bundle homomorphism $\mathcal{A} \otimes \mathcal{T} \rightarrow \mathcal{T}$, so $\mathcal{T}$ becomes a bundle of modules over the bundle $\mathcal{A}$ of Lie algebras. We will indicate the module structure simply by $(s, t) \mapsto s \bullet t$ if there is no risk of confusion. Note that by construction this defines a Lie algebra action, so

$$
\left\{s_{1}, s_{2}\right\} \bullet t=s_{1} \bullet\left(s_{2} \bullet t\right)-s_{2} \bullet\left(s_{1} \bullet t\right) .
$$

It is well known that there exists a unique element $E \in \mathfrak{g}$ called the grading element such that $[E, A]=j A$ holds for all elements $A \in \mathfrak{g}_{j}, j=-k, \ldots, k$. Clearly, $E$ is always contained in the center of $\mathfrak{g}_{0}$. This implies that $\operatorname{Ad}(b) \cdot E=E$ for each $b \in G_{0}$ and consequently $E$ acts by some scalar on each irreducible $G_{0^{-}}$ module. Now we can split the space $\mathbb{V}$ according to eigenvalues of the action of $E$, and we denote by $\mathbb{V}_{j}$ the component corresponding to the eigenvalue $j$. Then the action of $G_{0}$ maps each $\mathbb{V}_{j}$ to itself, while the (infinitesimal) action of $\mathfrak{g}_{i}$ maps $\mathbb{V}_{j}$ to $\mathbb{V}_{i+j}$ for each $i=-k, \ldots, k$.

Clearly, the decomposition $\mathbb{V}=\bigoplus_{j} \mathbb{V}_{j}$ is just $G_{0}$ invariant and not $P$ invariant. On the other hand, if we pass to the associated filtration by putting $\mathbb{V}^{j}:=\bigoplus_{j^{\prime} \geq j} \mathbb{V}_{j^{\prime}}$, then from the decomposition of $P$ in section 2.1 one concludes that this endows $\mathbb{V}$ with a $P$-invariant decreasing filtration. Note that if $\mathbb{V}$ is irreducible as a $\mathfrak{g}$-module, then it is generated as a module over the universal enveloping algebra by a highest weight vector, so the possible eigenvalues $j$ lie in the set $\left\{j_{0}-k: k \in \mathbb{N}\right\}$ where $j_{0}$ is eigenvalue of the highest weight vector. Thus, in the general (finite dimensional) case, the eigenvalues lie in the union of finitely many sets of that type (each such set corresponding to a $\mathfrak{g}$-irreducible component of $\mathbb{V}$ ). Passing to the associated bundles, we see that for each eigenvalue $j$ of $E$ on $\mathbb{V}$, we get a smooth subbundle $\mathcal{T}^{j} \subset \mathcal{T}$ corresponding to the $P$-submodule $\mathbb{V}^{j}$ of $\mathbb{V}$, and these subbundles form a decreasing filtration of $\mathcal{T}$.

2.5. Connections on tractor bundles. According to our definitions, there are many adjoint tractor bundles (and thus $\mathbb{V}$-tractor bundles for any $\mathbb{V}$ ) on a manifold $M$. As we will show below, the existence of a linear connection with certain properties is only possible on one (isomorphism class) of those, and this linear connection is equivalent to a normal Cartan connection on an adapted frame bundle $\mathcal{G} \rightarrow M$. Before we discuss this, we make some preliminary observations.

Let $\mathcal{T}$ be the $\mathbb{V}$-tractor bundle corresponding to $\mathcal{A}$ (and the adapted frame bundle $p: \mathcal{G} \rightarrow M)$. Let us denote by $\rho$ the effective $(\mathfrak{g}, P)$-representation on $\mathbb{V}$. By definition, $\mathcal{T}$ is an associated bundle to the principal bundle $\mathcal{G} \rightarrow M$, so we can identify smooth sections of $\mathcal{T}$ with smooth maps $\mathcal{G} \rightarrow \mathbb{V}$, which are $P$-equivariant. To write down this isomorphism explicitly, note first that the condition that $\mathcal{T}$ is associated to $\mathcal{G}$ implies that each point $u \in \mathcal{G}$ with $p(u)=x \in M$ gives rise to an 
isomorphism $\underline{u}: \mathbb{V} \rightarrow \mathcal{T}_{x}$, the fiber of $\mathcal{T}$ over $x$. Moreover, if $b \in P$ is any element, then $\underline{u \cdot b}(v)=\underline{u}(\rho(b)(v))$ for all $v \in \mathbb{V}$ where the dot denotes the principal right action of $P$ on $\mathcal{G}$.

If $t \in \Gamma(\mathcal{T})$ is a smooth section, then the corresponding function $\tilde{t}: \mathcal{G} \rightarrow \mathbb{V}$ is given by $\tilde{t}(u)=\underline{u}^{-1}(t(p(u)))$. By definition,

$$
\tilde{t}(u \cdot b)=\rho\left(b^{-1}\right)\left(\underline{u}^{-1}(t(p(u)))\right)=\rho\left(b^{-1}\right)(\tilde{t}(u))
$$

and so $\tilde{t}$ is seen to be equivariant. Conversely, the smooth section of $\mathcal{T}$ corresponding to a smooth equivariant map $\tau: \mathcal{G} \rightarrow \mathbb{V}$ is given by $x \mapsto \underline{u}(\tau(u))$, where $u \in \mathcal{G}$ is any point with $p(u)=x$. This is independent of the choice of $u$ since $\tau$ is equivariant.

Suppose that $\nabla$ is a linear connection on $\mathcal{T}$. Consider a point $u \in \mathcal{G}$ and a tangent vector $\xi \in T_{u} \mathcal{G}$ and let $x=p(u)$. For a smooth section $t \in \Gamma(\mathcal{T})$ we have a well defined element $\nabla_{T p \cdot \xi} t(x) \in \mathcal{T}_{x}$ and thus a point $\underline{u}^{-1}\left(\nabla_{T p \cdot \xi} t(x)\right) \in \mathbb{V}$. On the other hand, we also have the well defined element $\xi \cdot \tilde{t}(u) \in \mathbb{V}$. If $f$ is a smooth real valued function on $M$, then $\nabla_{T p \cdot \xi} f t(x)=t(x)(T p \cdot \xi) \cdot f(x)+f(x) \nabla_{T p \cdot \xi} t(x)$. On the other hand, $\tilde{f} t=\left(p^{*} f\right) \tilde{t}$ and thus $\xi \cdot \widetilde{f} t(u)=\xi \cdot\left(p^{*} f\right)(u) \tilde{t}(u)+\left(p^{*} f\right)(u) \xi \cdot \tilde{t}(u)$. But this implies that the difference $\underline{u}^{-1}\left(\nabla_{T p \cdot \xi} t(x)\right)-\xi \cdot \tilde{t}(u)$ depends only on $t(x)$ and thus only on $\tilde{t}(u)$. Hence $\xi$ induces a linear map $\Phi(\xi): \mathbb{V} \rightarrow \mathbb{V}$, which is characterized by the fact that $\underline{u}^{-1}\left(\nabla_{T p \cdot \xi} t(x)\right)-\xi \cdot \tilde{t}(u)=\Phi(\xi)(\tilde{t}(u))$, for each smooth section $t$ of $\mathcal{T}$.

Definition. (1) A linear connection $\nabla$ on $\mathcal{T}$ is called a $\mathfrak{g}$-connection if and only if for each tangent vector $\xi \in T_{u} \mathcal{G}$ the linear map $\Phi(\xi): \mathbb{V} \rightarrow \mathbb{V}$ defined above is given by the action of some element of $\mathfrak{g}$.

(2) A linear connection $\nabla$ on $\mathcal{T}$ is called non-degenerate if and only if for any point $x \in M$ and any nonzero tangent vector $\xi \in T_{x} M$ there exists a number $i$ and a (local) smooth section $t$ of $\mathcal{T}^{i}$ such that $\nabla_{T p \cdot \xi} t(x) \notin \mathcal{T}_{x}^{i}$.

(3) A tractor connection on $\mathcal{T}$ is a nondegenerate $\mathfrak{g}$-connection.

Note that the condition in the definition of a nondegenerate connection is of algebraic character: Since each $\mathcal{T}^{i}$ is a smooth subbundle of $\mathcal{T}$, we can form the quotient bundle $\mathcal{T} / \mathcal{T}^{i}$ and the projection $\pi_{i}: \mathcal{T} \rightarrow \mathcal{T} / \mathcal{T}^{i}$. For a smooth vector field $\xi$ on $M$ and a smooth section $s$ of $\mathcal{T}^{i}$, we can then consider the section $\pi_{i}\left(\nabla_{\xi} s\right)$ of $\mathcal{T} / \mathcal{T}^{i}$. Now one immediately verifies that the map $(\xi, s) \mapsto \pi_{i}\left(\nabla_{\xi} s\right)$ is bilinear over smooth functions on $M$, so it is induced by a vector bundle homomorphism $T M \otimes \mathcal{T}^{i} \rightarrow \mathcal{T} / \mathcal{T}^{i}$ and our condition says exactly that these bundle maps together separate points in $T M$.

For the special case of an adjoint tractor bundle, there is a simple and practical characterization of $\mathfrak{g}$-connections:

Proposition 2.6. A linear connection $\nabla$ on an adjoint tractor bundle $\mathcal{A} \rightarrow M$ is $a \mathfrak{g}$-connection if and only if it is compatible with the bracket, i.e. iff

$$
\nabla_{\xi}\left\{s_{1}, s_{2}\right\}=\left\{\nabla_{\xi} s_{1}, s_{2}\right\}+\left\{s_{1}, \nabla_{\xi} s_{2}\right\}
$$

holds for all smooth vector fields $\xi$ on $M$ and all sections $s_{1}, s_{2} \in \Gamma(\mathcal{A})$.

Proof. Let us first assume that $\nabla$ is a tractor connection. For sections $s_{1}, s_{2}$ of $\mathcal{A}$ with corresponding equivariant functions $\tilde{s}_{1}, \tilde{s}_{2}: \mathcal{G} \rightarrow \mathfrak{g}$, the function representing $\left\{s_{1}, s_{2}\right\}$ is just $u \mapsto\left[\tilde{s}_{1}(u), \tilde{s}_{2}(u)\right]$, the pointwise bracket in $\mathfrak{g}$. Since $\nabla$ is a tractor connection, we can compute the function representing $\nabla_{\xi} s$ as $u \mapsto$ $\bar{\xi} \cdot \tilde{s}(u)-[A(\bar{\xi}(u)), \tilde{s}(u)]$, where $\bar{\xi}$ is a lift of $\xi$ to a smooth vector field on $\mathcal{G}$ and 
each $A(\bar{\xi}(u))$ is an element of $\mathfrak{g}$. But then compatibility of the bracket follows immediately from the fact that $\bar{\xi}$ and $\left[A(\bar{\xi}(u)),{ }_{-}\right]$act as derivations with respect to the pointwise bracket.

Conversely, assume that $\nabla$ is compatible with the bracket. For a tangent vector $\xi \in T_{u} \mathcal{G}$ consider the map $\Phi(\xi): \mathfrak{g} \rightarrow \mathfrak{g}$. By definition, this map is characterized by $\underline{u}^{-1}\left(\nabla_{T p \cdot \xi} s(x)\right)=\xi \cdot \tilde{s}(u)+\Phi(\xi)(\tilde{s}(u))$ for each smooth section $s$ of $\mathcal{A}$. Now the fact that $\nabla$ is compatible with the bracket and the same is true for the first term on the right hand side immediately implies that $\Phi(\xi)$ is a derivation for each $\xi$. But since $\mathfrak{g}$ is semisimple, any derivation is inner, so the result follows.

Theorem 2.7. Let $\mathcal{A} \rightarrow M$ be an adjoint tractor bundle, $\mathcal{G} \rightarrow M$ an adapted frame bundle corresponding to a choice of a group $G$ with Lie algebra $\mathfrak{g}, \mathbb{V}$ an effective $(\mathfrak{g}, P)$-module and $\mathcal{T}$ the $\mathbb{V}$-tractor bundle for $\mathcal{A}$.

(1) A tractor connection $\nabla$ on $\mathcal{T}$ induces a Cartan connection $\omega$ on $\mathcal{G}$.

(2) Conversely, a Cartan connection $\omega$ on $\mathcal{G}$ induces tractor connections on all tractor bundles for $\mathcal{A}$.

Proof. (1) Let $u \in \mathcal{G}$ be a point and let $\xi \in T_{u} \mathcal{G}$ be a tangent vector. As before, we denote by $\rho$ both the representations of $P$ and $\mathfrak{g}$ on $\mathbb{V}$. Since $\nabla$ is a tractor connection on $\mathcal{T}$, we can find an element $\omega(\xi) \in \mathfrak{g}$ such that $\underline{u}^{-1}\left(\nabla_{T p \cdot \xi} t(x)\right)=$ $\xi \cdot \tilde{t}(u)+\rho(\omega(\xi))(\tilde{t}(u))$ holds for all smooth sections $t \in \Gamma(\mathcal{T})$. Moreover, since by assumption $\mathcal{T}$ corresponds to an effective $\mathfrak{g}$-module, the element $\omega(\xi)$ is uniquely determined by $\xi$, so we get a well defined map $\omega: T_{u} \mathcal{G} \rightarrow \mathfrak{g}$.

To see that $\omega: T \mathcal{G} \rightarrow \mathfrak{g}$ is smooth, it suffices to prove that for each smooth vector field $\xi$ on $\mathcal{G}$, the map $\mathcal{G} \rightarrow \mathfrak{g}$ given by $u \mapsto \omega(\xi(u))$ is smooth. For that, in turn, it suffices to show that $u \mapsto \rho(\omega(\xi(u)))(\tilde{t}(u))$ is smooth for each $t \in \Gamma(\mathcal{T})$. Now since $u \mapsto T p \cdot \xi(u)$ is a smooth map $\mathcal{G} \rightarrow T M$, the map $\mathcal{G} \rightarrow \mathbb{V}$ given by $u \mapsto \underline{u}^{-1}\left(\nabla_{T p \cdot \xi} t(p(u))\right)$ is smooth, too. Since $\xi \cdot \tilde{t}$ is obviously smooth, the result follows from the defining equation for $\omega$.

So it remains to verify the three defining conditions for a Cartan connection listed in 2.1. First, if $A \in \mathfrak{p}$ is any element, then the fundamental field $\zeta_{A}$ is vertical, i.e. $T p \cdot \zeta_{A}=0$. Thus, the defining equation for $\omega$ implies $\rho\left(\omega\left(\zeta_{A}\right)\right)(\tilde{t})=$ $-\zeta_{A} \cdot \tilde{t}$. But $\tilde{t}$ is equivariant, so $\tilde{t}(u \cdot b)=\rho\left(b^{-1}\right)(\tilde{t}(u))$. Putting $b=\exp (t A)$ in that formula and differentiating at $t=0$ we get $\zeta_{A} \cdot \tilde{t}(u)=-\rho(A)(\tilde{t}(u))$. Thus $\rho\left(\omega\left(\zeta_{A}\right)\right)(\tilde{t}(u))=\rho(A)(\tilde{t}(u))$ and since $\mathbb{V}$ is an effective $\mathfrak{g}$-module, this implies $\omega\left(\zeta_{A}\right)=A$. In particular, this implies that $\omega$ induces an isomorphism between the vertical tangent bundle of $\mathcal{G}$ and the trivial bundle $\mathcal{G} \times \mathfrak{p}$.

Next, we claim that $\omega$ restricts to a linear isomorphism on each tangent space. Since by assumption $\mathcal{G}$ and $\mathfrak{g}$ have the same dimension, it suffices to show that the restriction of $\omega$ to each tangent space $T_{u} \mathcal{G}$ is injective. So let $\xi \in T_{u} \mathcal{G}$ be any tangent vector. Let us first assume that $T p \cdot \xi \neq 0$. Then since $\nabla$ is a tractor connection, we can find a number $i$ and a smooth section $t \in \Gamma\left(\mathcal{T}^{i}\right)$ such that $\nabla_{T p \cdot \xi} t(x) \notin \mathcal{T}_{x}^{i}$. But, by the definition of $\omega$, we have $\nabla_{T p \cdot \xi} t(x)=\underline{u}(\xi \cdot \tilde{t}(u)+\rho(\omega(\xi))(\tilde{t}(u)))$. Now the function $\tilde{t}$ has values in $\mathbb{V}^{i}$, and so also $\xi \cdot \tilde{t}$ has values in $\mathbb{V}^{i}$. Thus, $\nabla_{T p \cdot \xi} t(x) \notin \mathcal{T}_{x}^{i}$ implies $\omega(\xi) \neq 0$. On the other hand, if $T p \cdot \xi=0$, then $\xi$ is vertical, and we have already shown that $\omega$ is injective on each vertical tangent space.

It remains to prove the equivariance of $\omega$. For $u \in \mathcal{G}, \xi \in T_{u} \mathcal{G}$ and $b \in P$ consider $\left(\left(r^{b}\right)^{*} \omega\right)(u)(\xi)$. By definition, this equals $\omega(u \cdot b)\left(\operatorname{Tr}^{b} \cdot \xi\right)$. Since the principal right action preserves the fibers of $\mathcal{G}$ we have $T p \cdot \operatorname{Tr}^{b} \cdot \xi=T p \cdot \xi$. Thus for a smooth 
section $t \in \Gamma(\mathcal{T})$ we compute

$$
\begin{aligned}
\rho\left(\omega(u \cdot b)\left(\operatorname{Tr}^{b} \cdot \xi\right)\right)(\tilde{t}(u \cdot b)) & =(\underline{u \cdot b})^{-1}\left(\nabla_{T p \cdot \xi} t(x)\right)-\left(\left(T r^{b} \cdot \xi\right) \cdot \tilde{t}\right)(u \cdot b) \\
& =\rho\left(b^{-1}\right)\left(\underline{u}^{-1}\left(\nabla_{T p \cdot \xi} t(x)\right)\right)-\xi \cdot\left(\tilde{t} \circ r^{b}\right)(u) .
\end{aligned}
$$

The equivariance of $\tilde{t}$ implies that $\tilde{t} \circ r^{b}=\rho\left(b^{-1}\right)(\tilde{t})$ and thus $\xi \cdot\left(\tilde{t} \circ r^{b}\right)(u)=$ $\rho\left(b^{-1}\right)(\xi \cdot \tilde{t}(u))$, and we get

$$
\begin{aligned}
\rho\left(\omega(u \cdot b)\left(\operatorname{Tr}^{b} \cdot \xi\right)\right) \tilde{t}(u \cdot b) & =\rho\left(b^{-1}\right)(\rho(\omega(u)(\xi)) \tilde{t}(u)) \\
& =\rho\left(\operatorname{Ad}\left(b^{-1}\right)(\omega(u)(\xi))\right) \tilde{t}(u \cdot b) .
\end{aligned}
$$

Again, the effectivity of $\rho$ implies that $\omega(u \cdot b)\left(T r^{b} \cdot \xi\right)=\operatorname{Ad}\left(b^{-1}\right)(\omega(u)(\xi))$.

(2) Let us conversely assume that $\omega$ is a Cartan connection on $\mathcal{G}$. For a smooth vector field $\xi$ on $M$ we can find a lift $\bar{\xi}$, which is a smooth vector field on $\mathcal{G}$. Then for a section $t$ of $\mathcal{T}$ with corresponding map $\tilde{t}: \mathcal{G} \rightarrow \mathbb{V}$ consider the map $\mathcal{G} \rightarrow \mathbb{V}$ defined by $\bar{\xi} \cdot \tilde{t}+\rho(\omega(\bar{\xi}))(\tilde{t})$. This map is obviously smooth and its value at a point $u$ depends only on the value of $\bar{\xi}$ at $u$ (and on $t$ ). Adding to $\bar{\xi}$ a fundamental vector field $\zeta_{A}$ does not change the expression since $\omega$ reproduces the generators of fundamental vector fields and $\tilde{t}$ is equivariant. These two facts show that the map does not depend on the choice of $\bar{\xi}$ but only on $\xi$. This in turn implies that we may choose $\bar{\xi}$ to be invariant under the principal right action of $P$, that is, $\operatorname{Tr}^{b} \cdot \bar{\xi}=\bar{\xi}$. Then both $\omega(\bar{\xi})$ and $\bar{\xi} \cdot \tilde{t}$ are $P$-equivariant and so the above function is also $P$ equivariant, since all its ingredients have that property. Thus, it defines a smooth section of $\mathcal{T}$ and we define $\nabla$ by letting $\nabla_{\xi} t$ be that section. One immediately verifies that this defines a linear connection on $\mathcal{T}$ and, by construction, this is a $\mathfrak{g}$-connection, so it remains to verify that $\nabla$ is nondegenerate.

Let us assume that $\xi \in T_{x} M$ is a nonzero tangent vector. Choose a point $u \in \mathcal{G}$ with $p(u)=x$ and a tangent vector $\bar{\xi} \in T_{u} \mathcal{G}$ with $T p \cdot \bar{\xi}=\xi$. Adding the value of an appropriate fundamental vector field to $\bar{\xi}$ we may without loss of generality assume that $\omega(\bar{\xi}) \in \mathfrak{g}_{-}=\mathfrak{g}_{-k} \oplus \cdots \oplus \mathfrak{g}_{-1}$, and clearly $\omega(\bar{\xi}) \neq 0$. Since $\mathbb{V}$ is an effective $\mathfrak{g}$-module, we can find a vector $v \in \mathbb{V}$ such that $\rho(\omega(\bar{\xi}))(v) \neq 0$. Without loss of generality we may assume that $v$ is an eigenvector for the grading element $E$, and we denote by $j$ its eigenvalue. Now we can find a smooth section $t$ of $\mathcal{T}^{j}$ such that $\underline{u}^{-1}(t(x))=v$. Then $\bar{\xi} \cdot \tilde{t}(u) \in \mathbb{V}^{j}$, so $\underline{u}^{-1}\left(\nabla_{\xi} t(x)\right)$ is congruent to $\rho(\omega(\bar{\xi}))(v)$ modulo $\mathbb{V}^{j}$. But $\rho(E)(\rho(\omega(\bar{\xi}))(v))=\rho(\omega(\bar{\xi}))(\rho(E)(v))+\rho([E, \omega(\bar{\xi})])(v)$, and since $\omega(\bar{\xi}) \in \mathfrak{g}_{-}$it follows immediately that expanding $\rho(\omega(\bar{\xi}))(v)$ as a sum of eigenvectors for $E$, smaller eigenvalues than $j$ have to occur. But this implies that $\nabla_{\xi} t(x) \notin \mathcal{T}_{x}^{j}$.

2.8. The fact that we can construct a Cartan connection from a tractor connection implies some relations between an adjoint tractor bundle admitting a tractor connection and other natural bundles. First consider the adjoint action of $P$ on $\mathfrak{g}$. This action leaves the subalgebra $\mathfrak{p}=\mathfrak{g}^{0}$ invariant, so we get an induced action on $\mathfrak{g} / \mathfrak{p}$. So we can form the associated bundle $\mathcal{G} \times_{P}(\mathfrak{g} / \mathfrak{p})$. Moreover, the projection $\mathfrak{g} \rightarrow \mathfrak{g} / \mathfrak{p}$ is by definition $P$-equivariant, so it induces a projection $\mathcal{A} \rightarrow \mathcal{G} \times{ }_{P}(\mathfrak{g} / \mathfrak{p})$ which gives an isomorphism $\mathcal{A} / \mathcal{A}^{0} \cong \mathcal{G} \times{ }_{P}(\mathfrak{g} / \mathfrak{p})$. On the other hand, using the Cartan connection it is easy to identify the bundle $\mathcal{G} \times_{P}(\mathfrak{g} / \mathfrak{p})$ : Consider the map $\mathcal{G} \times \mathfrak{g} \rightarrow T M$ which is given by $(u, A) \mapsto T p \cdot\left(\omega(u)^{-1}(A)\right)$. If $A$ lies in $\mathfrak{p}$, then $\omega(u)^{-1}(A)=\zeta_{A}$, which is a vertical field, so this map factors to a smooth map $\mathcal{G} \times(\mathfrak{g} / p) \rightarrow T M$. The equivariance of $\omega$ then immediately implies that this factors 
further to a homomorphism $\mathcal{G} \times_{P}(\mathfrak{g} / \mathfrak{p}) \rightarrow T M$ of vector bundles which covers the identity and is an isomorphism in each fiber, so it is a bundle isomorphism. Thus we see that we get a canonical projection $\Pi: \mathcal{A} \rightarrow T M$, which induces an isomorphism $\mathcal{A} / \mathcal{A}^{0} \cong T M$.

Let us next assume that we have fixed a $G$-invariant non-degenerate bilinear form $B$ on $\mathfrak{g}$. (It follows then automatically that $B$ is a nonzero multiple of the Killing form. For theoretical purposes we could restrict to the case where $B$ equals the Killing form, but for working with specific structure it is often preferable to use some multiple, so we prefer to keep this as an additional ingredient.) Then $B$ is also invariant under the action of $P$, and hence induces a nondegenerate bilinear form on $\mathcal{A}$. Moreover, it is easy to see from the grading that the Killing form (and thus also $B$ ) restricts to zero on $\mathfrak{g}_{p} \times \mathfrak{g}_{q}$ unless $p+q=0$. In particular, this implies that $B$ restricts to zero on $\mathfrak{p} \times \mathfrak{p}_{+}$, so it induces a non-degenerate pairing between $\mathfrak{g} / \mathfrak{p}$ and $\mathfrak{p}_{+}$, which is still invariant. In terms of the adjoint tractor bundle $\mathcal{A}$ this means that we get a duality between $\mathcal{A}^{1}$ and $\mathcal{A} / \mathcal{A}^{0} \cong T M$, which gives us an isomorphism $\mathcal{A}^{1} \cong T^{*} M$. Thus, the adjoint tractor bundle contains the cotangent bundle as a subbundle and has the tangent bundle as a quotient.

The isomorphism $T M \cong \mathcal{A} / \mathcal{A}^{0}$ leads to more structure on $T M$ : First, we get an induced filtration $T M=T^{-k} M \supset T^{-k+1} M \supset \cdots \supset T^{-1} M$, by putting $T^{i} M:=$ $\mathcal{A}^{i} / \mathcal{A}^{0} \subset \mathcal{A} / \mathcal{A}^{0}=T M$. By construction the rank of the smooth subbundle $T^{i} M \subset$ $T M$ equals the dimension of $\mathfrak{g}_{i} \oplus \cdots \oplus \mathfrak{g}_{-1}$. Moreover, if $\mathcal{G} \rightarrow M$ is an adapted frame bundle for $\mathcal{A}$ corresponding to a group $G$ with Lie algebra $\mathfrak{g}$, we consider the subgroups $G_{0} \leq P \leq G$ as in [2.1. We define $P_{+}=\exp \left(\mathfrak{p}_{+}\right) \leq P$ and consider $\mathcal{G}_{0}=\mathcal{G} / P_{+}$. It is easy to see that $\mathcal{G}_{0}$ is a principal bundle over $M$ with group $G_{0}$, see [6, 3.11]. Moreover, from the fact that $\mathcal{G}$ is a frame bundle for $\mathcal{A}$, one easily concludes that for each $i=-k, \ldots,-1$, the quotient bundle $T^{i} M / T^{i+1} M$ is the bundle associated to $\mathcal{G}_{0}$ with respect to the adjoint action of $G_{0}$ on $\mathfrak{g}_{i}$. Otherwise put, we can view $\mathcal{G}_{0}$ as a reduction of the associated graded vector bundle of the filtered vector bundle $T M$ to the structure group $G_{0}$. This reduction is the basic underlying geometric structure on $M$ which determines the parabolic geometry on $M$ in all cases except the two mentioned in 2.1 .

2.9. Curvature. The next step is to relate the curvature of a tractor connection with the curvature of the induced Cartan connection. The curvature of a linear connection $\nabla$ on $\mathcal{T}$ is defined as usual to be the $\operatorname{End}(\mathcal{T})$-valued two-form $R$ which is characterized by $R(\xi, \eta)(t(x))=\left(\nabla_{\xi} \nabla_{\eta}-\nabla_{\eta} \nabla_{\xi}-\nabla_{[\xi, \eta]}\right) t(x)$ for smooth vector fields $\xi, \eta$ and any smooth section $t$ of $\mathcal{T}$. We now show that this curvature is given by the action of the curvature of the induced Cartan connection. Recall (section 2.4) that we write $s \bullet t$ for the action of a section $s$ of $\mathcal{A}$ on a section $t$ of $\mathcal{T}$.

Proposition. Let $\nabla$ be a tractor connection on $\mathcal{T}$. Then there is an $\mathcal{A}$-valued two form $\kappa$ on $M$, such that $R(\xi, \eta)(t)=\kappa(\xi, \eta) \bullet t$ for all $t \in \mathcal{T}$. Moreover, if $\omega \in \Omega^{1}(\mathcal{G}, \mathfrak{g})$ is the Cartan connection induced by $\nabla$, then the function $\mathcal{G} \rightarrow \mathfrak{g}$ representing $\kappa(\xi, \eta)$ is $d \omega(\bar{\xi}, \bar{\eta})+[\omega(\bar{\xi}), \omega(\bar{\eta})]$, where $\bar{\xi}$ and $\bar{\eta}$ are lifts of $\xi$ and $\eta$ to smooth vector fields on $\mathcal{G}$.

Proof. To see that $d \omega(\bar{\xi}, \bar{\eta})+[\omega(\bar{\xi}), \omega(\bar{\eta})]$ depends only on $\xi$ and $\eta$ notice that we may check this at one point. So it suffices to see that the expression vanishes if one of the fields is vertical. Now for $A \in \mathfrak{p}$ let $i_{\zeta_{A}}$ be the insertion operator associated to the fundamental vector field $\zeta_{A}$. Since $i_{\zeta_{A}} \omega$ is constant, we have 
$i_{\zeta_{A}} d \omega=\mathcal{L}_{\zeta_{A}} \omega$, the Lie derivative, which equals $-\operatorname{ad}(A) \circ \omega$ by the equivariance of $\omega$. Consequently, $d \omega\left(\zeta_{A}, \bar{\eta}\right)=\left(i_{\zeta_{A}} d \omega\right)(\bar{\eta})=-[A, \omega(\bar{\eta})]$ which is what we had to show. Next, we claim that the function $d \omega(\bar{\xi}, \bar{\eta})+[\omega(\bar{\xi}), \omega(\bar{\eta})]: \mathcal{G} \rightarrow \mathfrak{g}$ is $P$ equivariant so $\kappa(\xi, \eta)$ is a well defined section of $\mathcal{A}$. Equivariancy of $\omega$ reads as $\omega(u \cdot b)\left(\operatorname{Tr}^{b} \cdot \bar{\xi}(u)\right)=\operatorname{Ad}\left(b^{-1}\right)(\omega(\bar{\xi}(u)))$. Applying the exterior derivative to the equation $\left(r^{b}\right)^{*} \omega=\operatorname{Ad}\left(b^{-1}\right) \circ \omega$ we get $\left(r^{b}\right)^{*} d \omega=\operatorname{Ad}\left(b^{-1}\right) \circ d \omega$, so

$$
d \omega(u \cdot b)\left(\operatorname{Tr}^{b} \cdot \bar{\xi}(u), \operatorname{Tr}^{b} \cdot \bar{\eta}(u)\right)=\operatorname{Ad}\left(b^{-1}\right)(d \omega(u)(\bar{\xi}(u), \bar{\eta}(u))) .
$$

Since $\bar{\xi}$ is a projectable vector field, we have $T p \cdot \bar{\xi}(u \cdot b)=T p \cdot \bar{\xi}(u)=T p \cdot \operatorname{Tr}^{b} \cdot \bar{\xi}(u)$ and similarly for $\bar{\eta}$. Thus we conclude the result from the first step of the proof.

To verify the formula for the curvature, let $t \in \Gamma(\mathcal{T})$ be a smooth section with corresponding smooth equivariant function $\tilde{t}: \mathcal{G} \rightarrow \mathbb{V}$ as in section 2.5 and let $\rho$ denote the representation of $(\mathfrak{g}, P)$ on $\mathbb{V}$. Then by definition of $\omega$, the function $\mathcal{G} \rightarrow \mathbb{V}$ representing $\nabla_{\eta} t \in \Gamma(\mathcal{T})$ is given by $\bar{\eta} \cdot \tilde{t}+\rho(\omega(\bar{\eta}))(\tilde{t})$. Thus $\nabla_{\xi} \nabla_{\eta} t$ is represented by the function

$$
\bar{\xi} \cdot \bar{\eta} \cdot \tilde{t}+\bar{\xi} \cdot \rho(\omega(\bar{\eta}))(\tilde{t})+\rho(\omega(\bar{\xi}))(\bar{\eta} \cdot \tilde{t})+\rho(\omega(\bar{\xi}))(\rho(\omega(\bar{\eta}))(\tilde{t})) .
$$

Now $\bar{\xi} \cdot \rho(\omega(\bar{\eta}))(\tilde{t})=\rho(\bar{\xi} \cdot \omega(\bar{\eta}))(\tilde{t})+\rho(\omega(\bar{\eta}))(\bar{\xi} \cdot \tilde{t})$. Using that $\bar{\xi} \cdot \bar{\eta} \cdot \tilde{t}-\bar{\eta} \cdot \bar{\xi} \cdot \tilde{t}=[\bar{\xi}, \bar{\eta}] \cdot \tilde{t}$ and that $\rho(\omega(\bar{\xi})) \circ \rho(\omega(\bar{\eta}))-\rho(\omega(\bar{\eta})) \circ \rho(\omega(\bar{\xi}))=\rho([\omega(\bar{\xi}), \omega(\bar{\eta})])$, we conclude that the section $\nabla_{\xi} \nabla_{\eta} t-\nabla_{\eta} \nabla_{\xi} t$ is represented by the function

$$
[\bar{\xi}, \bar{\eta}] \cdot \tilde{t}+\rho(\bar{\xi} \cdot \omega(\bar{\eta}))(\tilde{t})-\rho(\bar{\eta} \cdot \omega(\bar{\xi}))(\tilde{t})+\rho([\omega(\bar{\xi}), \omega(\bar{\eta})])(\tilde{t}) .
$$

Since $\bar{\xi}$ lifts $\xi$ and $\bar{\eta}$ lifts $\eta$, the bracket $[\bar{\xi}, \bar{\eta}]$ is a lift of the vector field $[\xi, \eta]$, and consequently the section $\nabla_{[\xi, \eta]} t$ is represented by the function

$$
[\bar{\xi}, \bar{\eta}] \cdot \tilde{t}+\rho(\omega([\bar{\xi}, \bar{\eta}]))(\tilde{t})
$$

Subtracting this from the above term we see that by definition of the exterior derivative $R(\xi, \eta)(t)$ is represented by the map $\rho(d \omega(\bar{\xi}, \bar{\eta})+[\omega(\bar{\xi}), \omega(\bar{\eta})])(\tilde{t})$.

2.10. Curvature normalization. The canonical Cartan connection for a parabolic geometry is characterized by a normalization condition on its curvature. To state this normalization condition, we have to reinterpret the curvature a little. In proposition 2.9 above, we have defined the $\mathcal{A}$-valued two form $\kappa$, which is by definition just the curvature of the induced Cartan connection. Now recall from section 2.8 that the cotangent bundle $T^{*} M$ is the associated bundle $\mathcal{G} \times{ }_{P} \mathfrak{p}_{+}$corresponding to the adjoint action of $P$ on $\mathfrak{p}_{+}=\mathfrak{g}^{1}$. Thus, $\kappa$ corresponds to a $P$-equivariant map $\tilde{\kappa}: \mathcal{G} \rightarrow \Lambda^{2} \mathfrak{p}_{+} \otimes \mathfrak{g}$. As we have observed, $\mathfrak{p}_{+}$is the dual $P$-module to $\mathfrak{g} / \mathfrak{p}$. The latter may be identified with $\mathfrak{g}_{-}=\mathfrak{g}_{-k} \oplus \cdots \oplus \mathfrak{g}_{-1}$ where the $P$-action on that space is given by applying the adjoint action and forgetting all terms which are not in $\mathfrak{g}_{-}$. Hence we can view $\tilde{\kappa}$ as an equivariant map from $\mathcal{G}$ to the space $L_{\text {alt }}^{2}\left(\mathfrak{g}_{-}, \mathfrak{g}\right)$ of bilinear skew symmetric maps. This form of $\tilde{\kappa}$ is usually called the curvature function of the Cartan connection $\omega$.

Since $\mathfrak{g} \cong \mathfrak{g}^{*}$ via $B$, we see that $L_{\text {alt }}^{2}\left(\mathfrak{g}_{-}, \mathfrak{g}\right)$ is dual (as a $G_{0}$-module) to $L_{\text {alt }}^{2}\left(\mathfrak{p}_{+}, \mathfrak{g}\right)$, and similarly, $L\left(\mathfrak{g}_{-}, \mathfrak{g}\right)$ is dual to $L\left(\mathfrak{p}_{+}, \mathfrak{g}\right)$. Since also $\mathfrak{p}_{+}$is a Lie subalgebra of $\mathfrak{g}$, we have the Lie algebra differential $L\left(\mathfrak{p}_{+}, \mathfrak{g}\right) \rightarrow L_{\text {alt }}^{2}\left(\mathfrak{p}_{+}, \mathfrak{g}\right)$, and its dual map can thus be viewed as a $G_{0}$-homomorphism $\partial^{*}: L_{\text {alt }}^{2}\left(\mathfrak{g}_{-}, \mathfrak{g}\right) \rightarrow L\left(\mathfrak{g}_{-}, \mathfrak{g}\right)$. Following Kostant (see [26]) one constructs inner products on the spaces in question such that $\partial$ and $\partial^{*}$ are adjoint operators, see $[6,2.5,2.6]$. In fact the map $\partial^{*}$ turns out to be a homomorphism of $P$-modules, see [6, 2.13] and is usually called the codifferential. To write down a formula for $\partial^{*}$, choose a basis $\left\{X_{\ell}\right\}$ of $\mathfrak{g}_{-}$and let $\left\{Z^{\ell}\right\}$ be the dual 
basis of $\mathfrak{p}_{+}$. Then for a bilinear alternating map $f: \mathfrak{g}_{-} \times \mathfrak{g}_{-} \rightarrow \mathfrak{g}$ and an element $X \in \mathfrak{g}_{-}$one has (see [33] section 5.1])

$$
\left(\partial^{*} f\right)(X)=\sum_{\ell}\left(\left[Z^{\ell}, f\left(X, X_{\ell}\right)\right]-\frac{1}{2} f\left(\left[Z^{\ell}, X\right]_{-}, X_{\ell}\right)\right) .
$$

Here $\left[Z^{\ell}, X\right]_{-}$denotes the $\mathfrak{g}_{-}$part of the bracket $\left[Z^{\ell}, X\right]$. Obviously, this expression is independent of the choice of the basis $\left\{X_{\ell}\right\}$.

A Cartan connection is called normal if and only if its curvature function $\tilde{\kappa}$ satisfies $\partial^{*} \circ \tilde{\kappa}=0$, i.e. iff $\tilde{\kappa}$ has $\partial^{*}$-closed values.

2.11. Curvature normalization for tractor connections. It is now quite easy to reformulate the normalization condition in a way which is appropriate for our purposes. If $\mathcal{A}$ is an adjoint tractor bundle on $M, p: \mathcal{G} \rightarrow M$ is an adapted frame bundle for $\mathcal{A}$ corresponding to some group $G, \mathcal{T}$ is any tractor bundle, and $\nabla$ is a tractor connection on $\mathcal{T}$, then by proposition 2.9, we have the $\mathcal{A}$-valued two form $\kappa$ describing the curvature of $\nabla$. Now by definition, $\kappa$ is a section of the bundle $\mathcal{G} \times{ }_{P} L_{\text {alt }}^{2}\left(\mathfrak{g}_{-}, \mathfrak{g}\right)$. Now since the codifferential $\partial^{*}: L_{\text {alt }}^{2}\left(\mathfrak{g}_{-}, \mathfrak{g}\right) \rightarrow L\left(\mathfrak{g}_{-}, \mathfrak{g}\right)$ is $P$-equivariant it induces a vector bundle homomorphism between the associated bundles, i.e. we have $\partial^{*}: \Lambda^{2} T^{*} M \otimes \mathcal{A} \rightarrow T^{*} M \otimes \mathcal{A}$, and we use the same symbol to denote the induced map on the spaces of smooth sections, i.e. $\partial^{*}: \Omega^{2}(M, \mathcal{A}) \rightarrow$ $\Omega^{1}(M, \mathcal{A})$. (In fact, a similar construction is possible for forms of higher degree, but we will not need this here.)

Definition. A tractor connection $\nabla$ on a tractor bundle $\mathcal{T}$ is called normal if and only if the form $\kappa \in \Omega^{2}(M, \mathcal{A})$ representing its curvature has the property that $\partial^{*}(\kappa)=0$.

By construction, if $\tilde{\kappa}: \mathcal{G} \rightarrow L_{\text {alt }}^{2}\left(\mathfrak{g}_{-}, \mathfrak{g}\right)$ is the function representing $\kappa$, then the function representing $\partial^{*}(\kappa)$ is just given by $\partial^{*} \circ \tilde{\kappa}$, so a tractor connection $\nabla$ on $\mathcal{T}$ is normal if and only if the induced Cartan connection on the adapted frame bundle is normal.

Using the formula for $\partial^{*}$ on the Lie algebra level from 2.10, one can easily compute the normality condition explicitly. To do this, we have to make another simple observation: As we have seen in 2.8, we have a natural projection $\Pi: \mathcal{A} \rightarrow$ $T M$ and dually a natural inclusion $T^{*} M \rightarrow \mathcal{A}^{*}$. The latter gives us inclusions $\Lambda^{2} T^{*} M \otimes \mathcal{A} \rightarrow \Lambda^{2} \mathcal{A}^{*} \otimes \mathcal{A}$ and $T^{*} M \otimes \mathcal{A} \rightarrow \mathcal{A}^{*} \otimes \mathcal{A}$. (Via the bilinear form $B$ from 2.8, could replace any of these occurrences of $\mathcal{A}^{*}$ by a $\mathcal{A}$.) In particular, we may extend the form $\kappa \in \Omega^{2}(M, \mathcal{A})$ naturally to a section of $\Lambda^{2} \mathcal{A}^{*} \otimes \mathcal{A}$, which has the property that it vanishes if either of its arguments lies in $\mathcal{A}^{0}$. We again denote this section by $\kappa$, and call it the tractor curvature of the tractor connection $\nabla$.

Now consider the formula for $\partial^{*}$ in 2.10. No change is made if we extend $f$ to be an alternating map on $\otimes^{2} \mathfrak{g}$ which annihilates $\mathfrak{p} \otimes \mathfrak{g}$. Then we may view $\partial^{*} f$ as a map from $(\mathfrak{g} / \mathfrak{p}) \rightarrow \mathfrak{g}$. That is we may then insert $X \in \mathfrak{g}$ into the defining expression and the result is zero if $X \in \mathfrak{p}$. In fact, since $f$ annihilates $\mathfrak{p} \otimes \mathfrak{g}$ (and so also $\mathfrak{g} \otimes \mathfrak{p}$ ), it is clear that we obtain the same answer, in any case, if we modify the formula by omitting the subscript minus in the bracket in the second term and summing over dual bases (with respect to $B$ ) for $\mathfrak{g}$ (since $f\left(-, X_{\ell}\right)$ is involved in both terms, so only the $\mathfrak{g}_{-}-$component of $X_{\ell}$ contributes). Bearing in mind that the Lie bracket of $\mathfrak{g}$ corresponds to the algebraic bracket on $\mathcal{A}$ we obtain the following formulation of the normalization condition. 
Proposition. Let $\kappa \in \Gamma\left(\Lambda^{2} \mathcal{A}^{*} \otimes \mathcal{A}\right)$ be the tractor curvature of a tractor connection $\nabla$ on some tractor bundle $\mathcal{T}$. Then $\nabla$ is normal if and only if the trace (with respect to $B$ ) over the first and last factor of the map $\otimes^{3} \mathcal{A} \rightarrow \mathcal{A}$ defined by

$$
\left(s_{1}, s_{2}, s_{3}\right) \mapsto\left\{s_{1}, \kappa\left(s_{2}, s_{3}\right)\right\}-\frac{1}{2} \kappa\left(\left\{s_{1}, s_{2}\right\}, s_{3}\right)
$$

vanishes.

2.12. Let us summarize the results we have obtained up to now and put them in perspective: Suppose that we start with the underlying geometric structure corresponding to some parabolic geometry. Suppose we can construct over each smooth manifold $M$ with that structure an adjoint tractor bundle $\mathcal{A}$ and a normal tractor connection on one of the tractor bundles for $\mathcal{A}$. Then on the appropriate adapted frame bundle $\mathcal{G}$ for $\mathcal{A}$ we get a normal Cartan connection. Thus we get an alternative approach to constructing the canonical Cartan connection for each of these underlying geometric structures.

It is known that $\mathcal{G}$ (and thus also $\mathcal{A}$, and each tractor bundle for $\mathcal{A}$ ) is uniquely determined up to isomorphism by the underlying geometric structure on $M$ and the fact that it carries a normal Cartan connection (see [6]). In that sense we can really speak about the normal tractor bundles on a manifold $M$ with an appropriate geometric structure.

The normal Cartan connection then induces a normal tractor connection on each of the tractor bundles for $\mathcal{A}$. These tractor connections depend only on the (canonical) bundle $\mathcal{G}$ and Cartan connection $\omega$, so they are invariant differential operators in any reasonable sense. We will show in the next section how to use these tractor connections to construct a much broader class of invariant differential operators.

\section{Fundamental $D$-Operators}

In this section we construct a basic set of invariant differential operators on a manifold equipped with a parabolic geometry. Throughout this section we will assume that we deal with a fixed parabolic geometry corresponding to a group $G$ with a $|k|$-grading of its Lie algebra $\mathfrak{g}$. As explained above each such manifold $M$ is equipped with a normal adjoint tractor bundle $\mathcal{A} \rightarrow M$ together with a normal tractor connection $\nabla^{\mathcal{A}}$ and a corresponding adapted frame bundle $\mathcal{G} \rightarrow M$. Then for any $(\mathfrak{g}, P)$-module $\mathbb{V}$ we have the corresponding (normal) $\mathbb{V}$-tractor bundle $\mathcal{T}=\mathcal{T}^{\mathbb{V}} \rightarrow M$ with a normal tractor connection $\nabla^{\mathcal{T}}$ on it.

For any $P$-module $\mathbb{W}$ the fundamental $D$-operator is a first order invariant differential operator from sections of $\mathcal{W}$ to sections of $\mathcal{A}^{*} \otimes \mathcal{W}$, where $\mathcal{W}$ is the associated bundle corresponding to $\mathbb{W}$. Such operators were first described for conformal structures in [18] and for CR structures in [19]. However in those cases the constructions were limited to the case that $\mathcal{W}$ was either a line bundle or the tensor product of a line bundle with a tractor bundle. Thus even for those structures we present here a considerable generalization as well as simultaneously treating all other parabolic geometries. (In fact there is an alternative approach to these operators which enables their construction in even wider circumstances. However we shall not need that construction here and it will be described elsewhere.)

3.1. The fundamental $D$-operators. Suppose that $\mathbb{W}$ is some representation of $P$ and $\mathcal{W}=\mathcal{G} \times{ }_{P} \mathbb{W}$ is the corresponding bundle. Then there is a bijective correspondence between the space $\Gamma(\mathcal{W})$ of smooth sections of $\mathcal{W}$ and the space 
$C^{\infty}(\mathcal{G}, \mathbb{W})^{P}$ of $P$-equivariant smooth maps from $\mathcal{G}$ to $\mathbb{W}$, see 2.5. Consider the exterior derivative $d: C^{\infty}(\mathcal{G}, \mathbb{W}) \rightarrow \Omega^{1}(\mathcal{G}, \mathbb{W})$ for $\mathbb{W}$ valued functions on $\mathcal{G}$. Via the Cartan connection $\omega$ on $\mathcal{G}$, we may identify $\Omega^{1}(\mathcal{G}, \mathbb{W})$ with the space $C^{\infty}\left(\mathcal{G}, \mathfrak{g}^{*} \otimes \mathbb{W}\right)$. Explicitly, this correspondence is given by mapping $\tau \in \Omega^{1}(\mathcal{G}, \mathbb{W})$ to the function $u \mapsto\left(A \mapsto \tau\left(\omega_{u}^{-1}(A)\right)\right)$, for $u \in \mathcal{G}$ and $A \in \mathfrak{g}$. Note that $C^{\infty}\left(\mathcal{G}, \mathfrak{g}^{*} \otimes \mathbb{W}\right)$ contains the subspace of $P$-equivariant smooth functions, which is isomorphic to $\Gamma\left(\mathcal{A}^{*} \otimes \mathcal{W}\right)$.

Proposition. (1) The exterior derivative $d: C^{\infty}(\mathcal{G}, \mathbb{W}) \rightarrow C^{\infty}\left(\mathcal{G}, \mathfrak{g}^{*} \otimes \mathbb{W}\right)$ maps $P_{-}$ equivariant functions to $P$-equivariant functions. Thus it restricts to an (obviously natural) first order differential operator $D=D^{\mathcal{W}}: \Gamma(\mathcal{W}) \rightarrow \Gamma\left(\mathcal{A}^{*} \otimes \mathcal{W}\right)$, the fundamental $D$-operator for the bundle $\mathcal{W}$.

(2) Suppose that $\mathbb{W}^{\prime}$ is another $P$-representation and that $\varphi: \mathbb{W} \rightarrow \mathbb{W}^{\prime}$ is a $P$-homomorphism. Denoting by $\varphi$ also the induced map $\Gamma(\mathcal{W}) \rightarrow \Gamma\left(\mathcal{W}^{\prime}\right)$ between sections of the associated bundles, we have $D^{\mathcal{W}^{\prime}} \varphi(s)=\varphi\left(D^{\mathcal{W}}(s)\right)$ for all smooth sections $s \in \Gamma(\mathcal{W})$.

(3) For the tensor product $\mathcal{W} \otimes \mathcal{W}^{\prime}$, we have the Leibniz rule, $D(s \otimes t)=D s \otimes$ $t+s \otimes D t$, for all smooth sections $s \in \Gamma(\mathcal{W})$ and $t \in \Gamma\left(\mathcal{W}^{\prime}\right)$.

Proof. For a one-form $\tau \in \Omega^{1}(\mathcal{G}, \mathbb{W})$ let us denote by $\tilde{\tau}: \mathcal{G} \rightarrow \mathfrak{g}^{*} \otimes \mathbb{W}$ the corresponding smooth function. Now consider an element $g \in P$ and the principal right action $r^{g}$ by $g$ on $\mathcal{G}$. By definition, we have

$$
\left(\widetilde{\left(r^{g}\right)^{*} \tau}\right)(u)(A)=\left(\left(r^{g}\right)^{*} \tau\right)(u)\left(\omega_{u}^{-1}(A)\right)=\tau(u \cdot g)\left(T r^{g} \cdot \omega_{u}^{-1}(A)\right) .
$$

Equivariancy of $\omega$ immediately implies that $\operatorname{Tr}^{g} \cdot \omega_{u}^{-1}(A)=\omega_{u \cdot g}^{-1}\left(\operatorname{Ad}\left(g^{-1}\right) \cdot A\right)$, so we can rewrite the equation as

$$
\left(\widetilde{\left(r^{g}\right)^{*} \tau}\right)(u)(A)=\tilde{\tau}(u \cdot g)\left(\operatorname{Ad}\left(g^{-1}\right) \cdot A\right)
$$

Now $f \in C^{\infty}(\mathcal{G}, \mathbb{W})$ is $P$-equivariant, if and only if $\left(r^{g}\right)^{*} f=\rho\left(g^{-1}\right) \circ f$, where $\rho$ denotes the representation of $P$ on $\mathbb{W}$. Applying the exterior derivative $d$ to this equation and keeping in mind that $d$ commutes with pullbacks and $\rho\left(g^{-1}\right)$ is linear, we get $\left(r^{g}\right)^{*} d f=\rho\left(g^{-1}\right) \circ d f$. Using this, we now compute

$$
\widetilde{d f}(u \cdot g)=\widetilde{\left(r^{g}\right)^{*} d f}(u) \circ \operatorname{Ad}(g)=\rho\left(g^{-1}\right) \circ \widetilde{d f}(u) \circ \operatorname{Ad}(g),
$$

which means exactly that $\widetilde{d f}$ is equivariant, too, so (1) is proved.

For $(2)$, one just has to notice that $\widetilde{\varphi(s)}=\varphi \circ \tilde{s}$, so linearity of $\varphi$ implies that $\widetilde{D \varphi(s)}=d \widetilde{\varphi(s)}=\varphi \circ d \tilde{s}=\widetilde{\varphi(D s)}$. Similarly, for (3) we have $\widetilde{s \otimes t}=\tilde{s} \otimes \tilde{t}$ and bilinearity of the pointwise tensor product implies the result.

The naturality of $D$ proved in (2) and (3) above justifies the notation $D$ for all the fundamental $D$-operators. Summarizing the result, we see that we are in a situation completely analogous to the case of covariant derivatives induced by a principal connection: For any associated bundle one has the fundamental $D$-operator $D: \Gamma(\mathcal{W}) \rightarrow \Gamma\left(\mathcal{A}^{*} \otimes \mathcal{W}\right)$ and these operators behave naturally with respect to all constructions with associated bundles. To emphasize the similarity to covariant derivatives, we will often write $D_{s} \sigma:=D \sigma(s)$ for $s \in \Gamma(\mathcal{A})$ and $\sigma \in \Gamma(\mathcal{W})$.

3.2. Although the above definition of the fundamental $D$-operators heavily involves the Cartan connection, we will next show that for a large class of bundles we can construct the fundamental $D$-operators directly from tractor connections 
on tractor bundles. First, we will compute the fundamental $D$-operators in two important special cases.

Proposition. (1) The fundamental D-operator for the trivial line bundle equals the extension of the exterior derivative, i.e. for $s \in \Gamma(\mathcal{A})$ and $f \in C^{\infty}(M, \mathbb{R})$ we have $D_{s} f=d f(\Pi(s))$, where $\Pi: \Gamma(\mathcal{A}) \rightarrow \Gamma(T M)$ is the canonical projection, see 2.8

(2) Let $\mathcal{T}$ be a tractor bundle and let $\nabla^{\mathcal{T}}$ be the normal tractor connection on $\mathcal{T}$. Then for smooth sections $s \in \Gamma(\mathcal{A})$ and $t \in \Gamma(\mathcal{T})$ we have $D_{s} t=\nabla_{\Pi(s)}^{\mathcal{I}} t-s \bullet t$, where $\bullet$ is the action introduced in 2.4.

Proof. (1) For $f \in C^{\infty}(M, \mathbb{R})$ the corresponding $P$-invariant function $\tilde{f}: \mathcal{G} \rightarrow \mathbb{R}$ is simply $p^{*} f=f \circ p$. Now $d p^{*} f=p^{*} d f$, and the result follows immediately.

(2) We know that the normal tractor connection on $\mathcal{T}$ is induced by the Cartan connection $\omega$ on $\mathcal{G}$ as described in the proof of theorem [2.7. For the smooth section $s \in \Gamma(\mathcal{A})$, consider the corresponding $P$-equivariant function $\tilde{s}: \mathcal{G} \rightarrow \mathfrak{g}$. Then the vector field $\Pi(s)$ corresponds exactly to the function $\pi \circ s$, where $\pi: \mathfrak{g} \rightarrow \mathfrak{g} / \mathfrak{p} \cong \mathfrak{g}_{-}$ is the canonical projection. Moreover, from the description of $T M$ as an associated bundle in 2.8, we see that $\Pi(s)(p(u))=T p \cdot \omega_{u}^{-1}(\tilde{s}(u))$. Hence $\omega_{u}^{-1}(\tilde{s}(u))$ is a lift of $\Pi(s)(p(u))$, so the proof of theorem 2.7(2) implies that

$$
\widetilde{\nabla_{\Pi(s)}^{\mathcal{T}} t}(u)=\omega_{u}^{-1}(\tilde{s}(u)) \cdot \tilde{t}+\rho(\tilde{s}(u))(\tilde{t}(u))=\widetilde{D_{s} t}(u)+\widetilde{s \bullet t}(u),
$$

and the result follows.

This result has several important consequences: First, proposition 3.1(3) and proposition 3.2 (1) imply that any fundamental $D$-operator satisfies a Leibniz rule with respect to multiplication by smooth functions, i.e. for $\sigma \in \Gamma(\mathcal{W}), s \in \Gamma(\mathcal{A})$ and $f \in C^{\infty}(M, \mathbb{R})$ we have $D_{s} f \sigma=d f(\Pi(s)) \sigma+f D_{s} \sigma$.

Second, for any bundle $\mathcal{W}$ associated to $\mathcal{G}$ we can consider the dual bundle $\mathcal{W}^{*}$. Denoting by $\langle\rangle:, \Gamma(\mathcal{W}) \times \Gamma\left(\mathcal{W}^{*}\right) \rightarrow C^{\infty}(M, \mathbb{R})$ the canonical pairing, we see from (3) of proposition 3.1 and (1) of proposition 3.2 that for $s \in \Gamma(\mathcal{A}), \sigma \in \Gamma(\mathcal{W})$ and $\sigma^{\prime} \in \Gamma\left(\mathcal{W}^{*}\right)$ we get

$$
d\left(\left\langle\sigma, \sigma^{\prime}\right\rangle\right)(\Pi(s))=\left\langle D_{s} \sigma, \sigma^{\prime}\right\rangle+\left\langle\sigma, D_{s} \sigma^{\prime}\right\rangle,
$$

so the fundamental $D$-operators behave similarly to covariant derivatives with respect to dual bundles.

Third, since the fundamental $D$-operators are defined for arbitrary natural bundles, they can clearly be iterated. Moreover, to compute iterates of a fundamental $D$-operator, the only thing one has to know, in addition to the fundamental $D$ operator on the bundle one starts with, is the normal tractor connection on the adjoint tractor bundle $\mathcal{A}$. Knowing this, we can compute the fundamental $D$ operator on $\mathcal{A}^{*} \cong \mathcal{A}$ using proposition [3.2 (2), and thus by proposition 3.1(3) we can compute the iterated fundamental $D$ 's.

As a variation on this theme, note that since both $D$ and $\nabla^{\mathcal{A}}$ satisfy a Leibniz rule, $t \otimes \sigma \mapsto \nabla_{\Pi(-)}^{\mathcal{A}} t \otimes \sigma+t \otimes D \sigma$ gives a well defined operator, so this also gives rise to an operator that can be iterated. An operator along these lines was used in [18] to produce a near complete invariant theory for even dimensional conformal structures. This provides evidence that our observations here provide a practical first step for parabolic invariant theory, see the next subsection. 
Finally note, that by proposition 3.1 (2) once we know the fundamental $D$ operator on any bundle $\mathcal{W}$, then we can compute the fundamental $D$-operator on any subbundle and on any quotient of $\mathcal{W}$. In particular, knowing the normal tractor connection on $\mathcal{A}$, we can compute the fundamental $D$-operators on the quotient $T M$ and the subbundle $T^{*} M$ and hence use proposition 3.1(3) on any tensor bundle. More generally, knowing the normal tractor connection on any tractor bundle $\mathcal{T}$, we can compute the fundamental $D$-operator on any subquotient of $\mathcal{T}$. In particular, if $\mathcal{T}^{i}$ is the filtration introduced in 2.4 then we can compute the fundamental $D$-operator on any irreducible component of $\mathcal{T}^{i} / \mathcal{T}^{i+1}$. The situation is further simplified by the fact that normal tractor connections behave well under constructions with vector bundles, i.e. knowing the normal tractor connection on $\mathcal{T}$ we also know the normal tractor connection on any exterior power and any symmetric power of $\mathcal{T}$, and so on.

3.3. An application. There has been considerable interest in the construction of polynomial invariants for parabolic structures [14, 15, 3, 24]. In these cases the authors are seeking local density-valued invariants which are in an appropriate sense polynomial in the jets of the structure. As described in, for example [3, 23, there are two steps involved in approaching this problem. The first is to represent the space of jets of the structure (modulo the action of diffeomorphisms) in an algebraically manageable manner. The second step involves describing how to use this algebraic structure to construct all invariants. An obvious approach for the first step is the Cartan connection (or tractor connection) acting on its curvature. However it is not known how to use the Cartan connection directly in this way. This led Fefferman and Graham [14, 15] to develop their ambient constructions to provide an alternative approach for $\mathrm{CR}$ and conformal structures. To the extent that these constructions are valid they capture the jet information (up to a given finite order) at a point in a section of a bundle corresponding to the $P$-submodule of a tensor-product of irreducible $G$-modules. However the constructions themselves are obstructed at finite order except in the case of odd dimensional conformal structures.

The fundamental $D$-operator provides another approach to this first step that works to all orders and is universal in that it deals with all parabolic structures.

Theorem. Let $\mathcal{W}$ be a natural bundle corresponding to a $P$-module $\mathbb{W}$ for some parabolic geometry, and let $k \in \mathbb{N}$ be any fixed order. Then for a section $\sigma$ of $\mathcal{W}$ the value of the section $\left(\sigma, D \sigma, D^{2} \sigma=D D \sigma, \ldots, D^{k} \sigma\right)$ of the natural bundle $\bigoplus_{i=0}^{k}\left(\otimes^{i} \mathcal{A}^{*} \otimes \mathcal{W}\right)$ at a point $x$ contains the full information of the $k$-jet of $\sigma$ at $x$. Moreover, to explicitly compute this section, it suffices to know the fundamental $D$-operator on $\mathcal{W}$ and the normal tractor connection on $\mathcal{A}$. If $\mathcal{W}$ is any subquotient of a tensor bundle or of a tensor power of $\mathcal{A}$, then knowledge of the normal tractor connection alone is sufficient. In particular, this applies to the tractor curvature $\kappa$, whose jets capture the jets of the structure itself.

Proof. The correspondence between sections of associated bundles and equivariant functions on the total space of the principal bundle also works at the level of $k$-jets. By the definition of the fundamental $D$-operators, knowing the value of $D \sigma$ at a point $x$ one can reconstruct the derivatives of the equivariant function $\mathcal{G} \rightarrow \mathbb{W}$ corresponding to $\sigma$ in all directions. Thus, $(\sigma(x), D \sigma(x))$ determines the one-jet of $\sigma$ in $x$. For $k$-jets the result then follows by induction. 
We have already observed in 3.2 above that to compute these iterated $D$ operators, it suffices to know the fundamental $D$-operator on $\mathcal{W}$ and the normal tractor connection on $\mathcal{A}$. That knowledge of the tractor connection alone suffices in the cases claimed follows immediately from the naturality results in proposition 3.1. The statement about the tractor curvature is clear, since $\kappa$ may be viewed as a section of $\Lambda^{2} \mathcal{A}^{*} \otimes \mathcal{A}$.

3.4. We have seen above that knowing the normal tractor connection on one tractor bundle allows us to compute the fundamental $D$-operator for a large class of bundles. Let us look more closely at the case of irreducible representations. On any irreducible representation of $P$, the nilpotent part $P_{+}$must act trivially, so any such representation is given by extending an irreducible representation of $G_{0}$ to $P$. On the Lie algebra level, we thus have to deal with irreducible representations of the (reductive) Lie algebra $\mathfrak{g}_{0}$. Now $\mathfrak{g}_{0}$ splits as a direct sum of its center $\mathfrak{z}\left(\mathfrak{g}_{0}\right)$ and its semisimple part $\mathfrak{g}_{0}^{s s}$. If $\mathbb{W}$ is any irreducible representation of $\mathfrak{g}_{0}$, then Schur's lemma implies that the center $\mathfrak{z}\left(\mathfrak{g}_{0}\right)$ acts by scalars, so there is a functional $w^{\prime}: \mathfrak{z}\left(\mathfrak{g}_{0}\right) \rightarrow \mathbb{K}$, where $\mathbb{K}=\mathbb{R}$ or $\mathbb{C}$, such that $A \cdot v=w(A) v$ for all $v \in \mathbb{V}$ and $A \in \mathfrak{z}\left(\mathfrak{g}_{0}\right)$. Now for any concrete choice of a $|k|$-graded Lie algebra $\mathfrak{g}$ it is easy to see that one needs just a few representations of $\mathfrak{g}$ in order to have any fundamental representation of $\mathfrak{g}_{0}^{s s}$ (with some action of the center) as a subquotient in the given representations of $\mathfrak{g}$. Hence, to deal efficiently with all irreducible representations, it essentially remains to deal with one-dimensional representations.

Before we come to that problem, let us make one more observation. Suppose that $\lambda$ is any representation of $P$ on a vector space $\mathbb{W}$ and let $\lambda^{\prime}$ be the infinitesimal representation of $\mathfrak{p}$. Then the kernel of $\lambda$ is a normal subgroup $Q$ of $P$, whose Lie algebra $\mathfrak{q}$ is exactly the kernel of $\lambda^{\prime}$. Since $Q$ is normal, $\mathfrak{q}$ is an ideal in $\mathfrak{p}$ and thus in particular stable under the adjoint action of $P$. In particular, this implies that we can form the associated bundle $\mathcal{Q}=\mathcal{G} \times{ }_{P} \mathfrak{q}$ which by construction is a subbundle of $\mathcal{A}^{0}$ and hence also of $\mathcal{A}$. Now if $\mathcal{W}$ is the associated bundle corresponding to $\mathbb{W}$, $\sigma \in \Gamma(\mathcal{W})$ is a smooth section and $\tilde{\sigma}: \mathcal{G} \rightarrow \mathbb{W}$ is the corresponding $P$-equivariant smooth function, then by construction $\tilde{\sigma}$ is $Q$-invariant. Infinitesimally, this means that $\xi \cdot \tilde{\sigma}=0$ for any tangent vector $\xi$ on $\mathcal{G}$ such that $\omega(\xi) \in \mathfrak{q}$. But this immediately implies that for a smooth section $s$ of $\mathcal{Q} \subset \mathcal{A}$, we have $D_{s} \sigma=0$, so $D \sigma$ actually is a smooth section of $(\mathcal{A} / \mathcal{Q})^{*} \otimes \mathcal{W}$.

3.5. Natural line bundles. The actual construction of fundamental $D$-operators for line bundles depends on the structure in question, so we just outline some basic results here. For simplicity, we restrict to the case of real line bundles here, complex line bundles can be dealt with similarly. In section 4 below, we will give a uniform treatment of these operators in the case of irreducible parabolic geometries. It should be noted that one can always get some natural line bundles as subbundles of tractor bundles, so the methods outlined in 3.2 are applicable to these bundles. In fact, suppose that $\mathcal{T}$ is a tractor bundle and $\mathcal{T}^{i}$ is the smallest component (the component with $i$ maximal) in the filtration from 2.4. Then by definition of the filtration, this corresponds to a completely reducible representation of $P$. Let $\ell$ be the rank of $\mathcal{T}^{i}$, and consider the $\ell$-th exterior power $\Lambda^{\ell} \mathcal{T}$ of $\mathcal{T}$, which is again a tractor bundle, whose normal tractor connection is known, once the normal tractor connection on $\mathcal{T}$ is known. But now it is obvious that the smallest component in the filtration of $\Lambda^{\ell} \mathcal{T}$ is just $\Lambda^{\ell} \mathcal{T}^{i}$ and this is one dimensional. Finally, it is easy to see that the center of $\mathfrak{g}_{0}$ always acts nontrivially on the representation corresponding 
to $\mathcal{T}^{i}$ (in particular, this is easy to see for the grading element, see 2.4), so we really obtain a nontrivial line bundle in that way. Moreover, it is worth noting that the tensor product makes the set of line bundles associated to $\mathcal{G}$ into an Abelian group, and on the level of functionals $\mathfrak{z}\left(\mathfrak{g}_{0}\right) \rightarrow \mathbb{K}^{*}$ the tensor product corresponds to the sum of functionals.

First we establish some notation. Recall that in the identification of $T^{*} M$ with an associated bundle we have used a $G$-invariant bilinear form $B$ on $\mathfrak{g}$. Let us also denote by $B$ the corresponding map $\Gamma(\mathcal{A}) \times \Gamma(\mathcal{A}) \rightarrow C^{\infty}(M, \mathbb{R})$. We will consider the fundamental $D$-operator on a line bundle induced from a representation $\tilde{w}$ of $P$ on $\mathbb{R}$ such that $Q:=\operatorname{ker}(\tilde{w})$ satisfies $\operatorname{dim}(Q)=\operatorname{dim}(P)-1$. We write $w$ for the corresponding representation of $\mathfrak{p}$. We will denote the one-dimensional real representation corresponding to $\tilde{w}$ by $\mathbb{R}[\tilde{w}]$. The corresponding line bundle will be denoted by $\mathcal{E}[\tilde{w}]$.

Proposition. (1) Given any non-vanishing (local) section $\sigma \in \Gamma(\mathcal{E}[\tilde{w}])$ there is a corresponding unique (local) smooth section $A^{\sigma}$ of $\mathcal{A}^{0}$ such that $D_{s} \sigma=B\left(A^{\sigma}, s\right) \sigma$ for all $s \in \Gamma(\mathcal{A})$. Moreover, $B\left(A^{\sigma}, A^{\sigma}\right)$ is constant.

(2) For any section $\sigma^{\prime}$ of $\mathcal{E}[\tilde{w}]$ and $\sigma$ as in (1) consider the smooth function $f=\sigma^{\prime} \sigma^{-1}: M \rightarrow \mathbb{R}$. Then $D_{s} \sigma^{\prime}=B\left(A^{\sigma}, s\right) \sigma^{\prime}+d f(\Pi(s)) \sigma$.

(3) Suppose that $\alpha$ is any real number such that $\tilde{w}^{\alpha}$ makes sense as a representation of $P$ and $\sigma$ is any (local) section of $\mathcal{E}[\tilde{w}]$. Then $\sigma^{\alpha}$ is a section of $\mathcal{E}\left[\tilde{w}^{\alpha}\right]$ and we have $D_{s}\left(\sigma^{\alpha}\right)=\alpha \sigma^{\alpha-1} D_{s} \sigma$. Moreover, if $\sigma$ is non-vanishing, then $A^{\sigma^{\alpha}}=\alpha A^{\sigma}$.

Proof. (1) Since $\sigma$ is non-vanishing, we can form $\sigma^{-1} D \sigma$, which is a section of $\mathcal{A}^{*}$. Since $B$ is non-degenerate, any such section may be represented as $s \mapsto B\left(A^{\sigma}, s\right)$ for a unique section $A^{\sigma}$ of $\mathcal{A}$. So to prove (1) we only have to show that $A^{\sigma}$ is actually a section of $\mathcal{A}^{0}$ and that $B\left(A^{\sigma}, A^{\sigma}\right)$ is constant. But from 3.4 we know that $D_{s} \sigma=0$ for $s \in \Gamma(\mathcal{Q})$ where $\mathcal{Q}$ is the bundle corresponding to the kernel of $w$. In particular, $\mathcal{A}^{1} \subset \mathcal{Q}$, so $B\left(A^{\sigma}, s\right)=0$ for $s \in \Gamma\left(\mathcal{A}^{1}\right)$. But this already shows that $A^{\sigma} \in \Gamma\left(\mathcal{A}^{0}\right)$, since the pairing between $\mathcal{A} / \mathcal{A}^{0}$ and $\mathcal{A}^{1}$ induced by $B$ is non-degenerate, see 2.8. Next, if $s \in \Gamma\left(\mathcal{A}^{0}\right)$, then from the definitions of the fundamental $D$-operator and of $A^{\sigma}$ it follows immediately that $B\left(A^{\sigma}, s\right)=-w(s)$, where we also use $w$ to denote the map $\Gamma\left(A^{0}\right) \rightarrow C^{\infty}(M, \mathbb{R})$ induced by $w: \mathfrak{p} \rightarrow \mathbb{R}$. Now consider the map $\widetilde{A^{\sigma}}: \mathcal{G} \rightarrow \mathfrak{p}$ corresponding to $A^{\sigma}$. Since the Killing form vanishes on $\mathfrak{p} \times \mathfrak{p}_{+}$, the function $B\left(A^{\sigma}, A^{\sigma}\right)$ depends only on the component of $\widetilde{A^{\sigma}}$ in $\mathfrak{g}_{0}$. On the other hand, since the Killing form is non-degenerate on $\mathfrak{g}_{0} \times \mathfrak{g}_{0}$, there is a unique element $A \in \mathfrak{g}_{0}$ such that $w(C)=-B(A, C)$ for all $C \in \mathfrak{p}$. Now since $B\left(A^{\sigma}, s\right)$ corresponds to the function $u \mapsto B\left(\widetilde{A^{\sigma}}(u), \tilde{s}(u)\right)$, we see from the above that the $\mathfrak{g}_{0}$-component of $\widetilde{A^{\sigma}}$ must be constant and equal to $A$, so also $B\left(A^{\sigma}, A^{\sigma}\right)$ is constant.

(2) We have $\sigma^{\prime}=f \sigma$, so the Leibniz rule from 3.2 implies that $D_{s} \sigma^{\prime}=d f(\Pi(s)) \sigma$ $+f D_{s} \sigma$, and rewriting the last term as $f B\left(A^{\sigma}, s\right) \sigma$ the result follows.

(3) If $\sigma$ corresponds to the function $\tilde{\sigma}: \mathcal{G} \rightarrow \mathbb{R}$, then $\tilde{\sigma}^{\alpha}$ is equivariant for the action $\tilde{w}^{\alpha}$, so it defines a smooth section of the bundle $\mathcal{E}\left[\tilde{w}^{\alpha}\right]$. But then for $A \in \mathfrak{g}$, we clearly get $\omega_{u}^{-1}(A) \cdot \sigma^{\alpha}=\alpha \sigma^{\alpha-1} \omega_{u}^{-1}(A) \cdot \sigma$, so the formula for $D_{s} \sigma^{\alpha}$ follows. If in addition $\sigma$ is non-vanishing, then so is $\sigma^{\alpha}$ and by (1) we have

$$
B\left(A^{\sigma^{\alpha}}, s\right) \sigma^{\alpha}=D_{s} \sigma^{\alpha}=\alpha \sigma^{\alpha-1} D_{s} \sigma=\alpha B\left(A^{\sigma}, s\right) \sigma^{\alpha},
$$

and the result follows. 
Note that viewing $D$ as having values in sections of $\mathcal{A} \otimes \mathcal{W}$ rather than $\mathcal{A}^{*} \otimes \mathcal{W}$, part (1) and (2) can be rewritten as $D \sigma=A^{\sigma} \otimes \sigma$ for $\sigma$ non-vanishing and $D \sigma^{\prime}=$ $I(d f) \otimes \sigma+A^{\sigma} \otimes \sigma^{\prime}$ with $f=\sigma^{-1} \sigma^{\prime}$ and $I$ denoting the inclusion $T^{*} M \rightarrow \mathcal{A}$ from 2.8.

Note further that the last part of the above proposition together with the fact that the tensor product of line bundles corresponds to the sum of functionals implies that it suffices to compute the fundamental $D$-operators for line bundles corresponding to a basis of the space of linear functionals on $\mathfrak{z}\left(\mathfrak{g}_{0}\right)$. This observation is nontrivial since it may well occur that $\mathcal{E}\left[\tilde{w}^{\alpha}\right]$ makes sense for all $\alpha \in \mathbb{R}$, see 4.15

3.6. The Lie bracket on adjoint tractors. As we have outlined, the philosophy of the fundamental $D$-operators is to view the adjoint tractor bundle $\mathcal{A}$ as a replacement for the tangent bundle and the fundamental $D$-operators as a replacement for covariant derivatives. From this point of view, it is desirable to have objects analogous to the torsion and curvature of covariant derivatives. The main problem in constructing such analogues is that we have not yet an analogue of the Lie bracket of vector fields on the level of adjoint tractors. Such an analogue can however be constructed using the following observation: By definition, sections of the adjoint tractor bundle $\mathcal{A}$ are in bijective correspondence with $P$-equivariant smooth functions $\mathcal{G} \rightarrow \mathfrak{g}$. Now if $\tilde{s}: \mathcal{G} \rightarrow \mathfrak{g}$ is such a function, then we can associate to it a vector field $\xi \in \mathfrak{X}(\mathcal{G})$ uniquely characterized by $\omega(\xi(u))=\tilde{s}(u)$. Moreover, equivariancy of $\omega$, which can be written as $\omega\left(T r^{g} \cdot \xi(u)\right)=\operatorname{Ad}\left(g^{-1}\right)(\omega(\xi(u)))$, and equivariancy of $\tilde{s}$ imply that the vector field $\xi$ is right invariant, i.e. $\left(r^{g}\right)^{*} \xi=\xi$ or $\xi(u \cdot g)=\operatorname{Tr}^{g} \cdot \xi(u)$. On the other hand, if $\xi$ is a right invariant vector field on $\mathcal{G}$, then $\omega(\xi): \mathcal{G} \rightarrow \mathfrak{g}$ is a $P$-equivariant smooth function, so we actually get a bijective correspondence between smooth sections of $\mathcal{A}$ and right invariant vector fields on $\mathcal{G}$.

Now it is an elementary observation that the Lie bracket of two right invariant vector fields is again right invariant, so by this construction we get a Lie bracket on the space $\Gamma(\mathcal{A})$ of smooth sections of the adjoint tractor bundle. Fortunately, this bracket can be computed easily in terms of fundamental $D$-operators:

Proposition. Consider the fundamental D-operator on sections of $\mathcal{A}$, let $\Pi$ : $\Gamma(\mathcal{A}) \rightarrow \mathfrak{X}(M)$ be the canonical projection, and let $\kappa \in \Gamma\left(\Lambda^{2} \mathcal{A}^{*} \otimes \mathcal{A}\right)$ be the tractor curvature of the tractor connection on $\mathcal{A}$. Then for $s_{1}, s_{2} \in \Gamma(\mathcal{A})$ the Lie bracket described above is given by

$$
\left[s_{1}, s_{2}\right]=D_{s_{1}} s_{2}-D_{s_{2}} s_{1}-\kappa\left(s_{1}, s_{2}\right)+\left\{s_{1}, s_{2}\right\} .
$$

Moreover, the Lie bracket has the following properties:

(1) $\Pi\left(\left[s_{1}, s_{2}\right]\right)=\left[\Pi\left(s_{1}\right), \Pi\left(s_{2}\right)\right]$, where the second bracket is the Lie bracket of vector fields on $M$.

(2) $\left[s_{1}, f s_{2}\right]=\left(\Pi\left(s_{1}\right) \cdot f\right) s_{2}+f\left[s_{1}, s_{2}\right]$ for all $f \in C^{\infty}(M, \mathbb{R})$.

Proof. Let $\tilde{s}_{1}, \tilde{s}_{2}: \mathcal{G} \rightarrow \mathfrak{g}$ be the $P$-equivariant functions corresponding to $s_{1}, s_{2} \in$ $\Gamma(\mathcal{A})$, and let $\xi_{1}, \xi_{2}$ be the corresponding right invariant vector fields on $\mathcal{G}$. The bracket $\left[s_{1}, s_{2}\right]$ is then defined by $\widetilde{\left.s_{1}, s_{2}\right]}=\omega\left(\left[\xi_{1}, \xi_{2}\right]\right)$. Inserting the definition of the exterior derivative and the definition of the curvature function $\tilde{\kappa}$ (see 2.10) of the Cartan connection $\omega$, we get

$$
\widetilde{\left[s_{1}, s_{2}\right]}=\xi_{1} \cdot \omega\left(\xi_{2}\right)-\xi_{2} \cdot \omega\left(\xi_{1}\right)-\tilde{\kappa}\left(\omega\left(\xi_{1}\right), \omega\left(\xi_{2}\right)\right)+\left[\omega\left(\xi_{1}\right), \omega\left(\xi_{2}\right)\right],
$$


where the last bracket is in $\mathfrak{g}$. By definition, $\omega\left(\xi_{i}\right)=\tilde{s}_{i}$, so the definition of the fundamental $D$-operators implies that the first two terms correspond to $D_{s_{1}} s_{2}-$ $D_{s_{2}} s_{1}$, while the last term exactly represents $\left\{s_{1}, s_{2}\right\}$. Finally, proposition [2.9 immediately implies that the remaining term exactly represents $\kappa\left(s_{1}, s_{2}\right)$.

Next, the identification of $T M$ with a bundle associated to $\mathcal{G}$ from 2.8 implies that the vector field $\xi_{i}$ is a lift of the vector field $\Pi\left(s_{i}\right)$, so the Lie bracket $\left[\xi_{1}, \xi_{2}\right]$ is a lift of $\left[\Pi\left(s_{1}\right), \Pi\left(s_{2}\right)\right]$, which implies (1).

Finally, for $(2)$ we just have to observe that $\widetilde{\left(f s_{2}\right)}=(f \circ p) \tilde{s}_{2}$, so the right invariant vector field on $\mathcal{G}$ corresponding to $f s_{2}$ is given by $(f \circ p) \xi_{2}$. But then $\left[\xi_{1},(f \circ p) \xi_{2}\right]=\left(\xi_{1} \cdot(f \circ p)\right) \xi_{2}+(f \circ p)\left[\xi_{1}, \xi_{2}\right]$, so $(2)$ follows since $\xi_{1} \cdot(f \circ p)=$ $\left(\left(T p \cdot \xi_{1}\right) \cdot f\right) \circ p$.

Remark. The proposition above also shows that viewing the fundamental $D^{-}$ operator on $\mathcal{A}$ as an analogue of an affine connection, then the obvious analogue $D_{s_{1}} s_{2}-D_{s_{2}} s_{1}-\left[s_{1}, s_{2}\right]$ of the torsion is simply given by $\kappa\left(s_{1}, s_{2}\right)-\left\{s_{1}, s_{2}\right\}$.

3.7. To finish this section, we want to discuss curvature-like properties of the fundamental $D$-operators. First we have to note that for any bundle $\mathcal{W}$ the obvious analogue

$$
\left(s_{1}, s_{2}, t\right) \mapsto D_{s_{1}} D_{s_{2}} t-D_{s_{2}} D_{s_{1}} t-D_{\left[s_{1}, s_{2}\right]} t
$$

of curvature vanishes. Indeed, if $\tilde{t}: \mathcal{G} \rightarrow \mathbb{W}$ is the function corresponding to $t$ and $\xi_{i}$ are the right invariant vector fields corresponding to the smooth sections $s_{i}$ of $\mathcal{A}$, then the above expression is represented by the function $\xi_{1} \cdot \xi_{2} \cdot \tilde{t}-\xi_{2}$. $\xi_{1} \cdot \tilde{t}-\left[\xi_{1}, \xi_{2}\right] \cdot \tilde{t}=0$. This is not surprising, since essentially the fundamental $D$ operator was constructed from a trivialization of $T \mathcal{G}$. Nevertheless, we still get that the alternation of $D D t$ can be expressed by lower order terms involving curvature. This can be expressed as follows:

Proposition. Let $\mathcal{W}$ be any bundle associated to $\mathcal{G}$, and let $\kappa$ be the tractor curvature of the tractor connection on $\mathcal{A}$. Then for smooth sections $s_{1}, s_{2}$ of $\mathcal{A}$ and $t$ of $\mathcal{W}$ we have

$$
D D t\left(s_{1}, s_{2}\right)-D D t\left(s_{2}, s_{1}\right)=-D_{\kappa\left(s_{1}, s_{2}\right)} t+D_{\left\{s_{1}, s_{2}\right\}} t .
$$

Proof. The flatness of the fundamental $D$-operators observed above can be expressed as $D_{s_{1}} D_{s_{2}} t-D_{s_{2}} D_{s_{1}} t=D_{\left[s_{1}, s_{2}\right]} t$. From proposition 3.6 we know that $\left[s_{1}, s_{2}\right]=D_{s_{1}} s_{2}-D_{s_{2}} s_{1}-\kappa\left(s_{1}, s_{2}\right)+\left\{s_{1}, s_{2}\right\}$. Now we just have to observe that applying the naturality results from proposition 3.1 (2) and (3) to the map $s_{2} \otimes D t \mapsto D_{s_{2}} t$, we get $D D t\left(s_{1}, s_{2}\right)=D_{s_{1}} D_{s_{2}} t-D_{D_{s_{1}} s_{2}} t$ and the result follows.

Remarks. (1) The result of this proposition may look slightly strange since it seems that the tractor curvature $\kappa$ acts differentially on $t$ instead of algebraically as one would expect. One has however to keep in mind that the curvature of a Cartan connection contains a torsion part (the part with values in $\mathfrak{g}_{-}$) and a part with values in $\mathfrak{p}$, which is the analogue of the curvature of a linear connection. By definition of the fundamental $D$-operators, the $\mathfrak{p}$-part actually acts algebraically while the torsion part really acts differentially, so the behavior is as expected.

(2) A particularly nice consequence of the above proposition is that if one considers the sequence $(t, D t, D D t, \ldots)$ for a smooth section $t \in \Gamma(\mathcal{W})$, then this encodes 
not only the infinite jet of the section $t$ but also the actions of jets of the curvature on jets of $t$. This should be especially relevant when dealing with questions of invariants.

(3) Since the normal Cartan connection gives a canonical trivialization of $T \mathcal{G}$ it determines a linear connection on $T \mathcal{G}$. Since this is just the exterior derivative in the trivialization it is, of course, curvature flat. On the other hand it does have torsion and in the trivialization this is given by $\tilde{\kappa}(-,)-,\left[-,{ }_{-}\right]$. Restricting to right invariant vector fields enables us to interpret this picture via the tractor bundles and this is another way of viewing the results above.

\section{Adjoint traCtor BUNDLES FOR IRREDUCIBLE PARABOliC GEOMETRIES}

In this section we construct the normal adjoint tractor bundle for any manifold equipped with a parabolic geometry corresponding to a $|1|$-grading $\mathfrak{g}=\mathfrak{g}_{-1} \oplus \mathfrak{g}_{0} \oplus \mathfrak{g}_{1}$ on a simple Lie algebra $\mathfrak{g}$. Since $|1|$-gradings are characterized among all $|k|-$ gradings by the fact that the tangent bundle corresponds to an irreducible representation of $\mathfrak{p}$, we call these geometries irreducible parabolic geometries. Another name for these structures, which is frequently used in the literature, is almost Hermitian symmetric- or AHS-structures.

The construction of the canonical Cartan connection for this class of structures has quite a long history. In several cases a construction was already given by Cartan, see e.g. [10]. More recent presentations can be found for example in [30] and [8, part II]. Also, tractor calculi for several of these structures have been known for quite some time, see e.g. [32] and 2] (conformal and projective), and 21] for the case of almost Grassmannian structures. Tractor bundles and connections (corresponding to standard or in some cases spinor representations) for all such structures were constructed (case by case) in [4, part I]. Via a construction which treats almost all the geometries in a uniform manner, we present here, for the first time, a simultaneous construction of tractor calculi for the entire class of irreducible parabolic geometries.

4.1. Let $\mathfrak{g}=\mathfrak{g}_{-1} \oplus \mathfrak{g}_{0} \oplus \mathfrak{g}_{1}$ be a real $|1|$-graded simple Lie algebra of rank bigger than two (which excludes the case of one-dimensional projective structures, which is a degenerate case). We say that $\mathfrak{g}$ is of projective type if either $\mathfrak{g}$ or its complexification is isomorphic to some $\mathfrak{s l}(n, \mathbb{C})$ with the $|1|$-grading corresponding to the first or last simple root. Let $G$ be a Lie group with Lie algebra $\mathfrak{g}$, and let $G_{0} \subset P \subset G$ be the subgroups as introduced in 2.1 The restriction of the adjoint action to $\mathfrak{g}_{-1}$ gives rise to a homomorphism Ad : $G_{0} \rightarrow G L\left(\mathfrak{g}_{-1}\right)$. If $\mathfrak{g}$ is not of projective type, then an irreducible parabolic geometry of type $(G, P)$ on a smooth manifold $M$ with $\operatorname{dim}(M)=\operatorname{dim}\left(\mathfrak{g}_{-1}\right)$ is then simply given by a principal $G_{0}$-bundle $p: \mathcal{G}_{0} \rightarrow M$ which is a reduction (corresponding to the above homomorphism) of the frame bundle $\mathcal{P} M$ of $M$. Equivalently, we must have a one-form $\theta \in \Omega^{1}\left(\mathcal{G}_{0}, \mathfrak{g}_{-1}\right)$ which is strictly horizontal, i.e. $\operatorname{ker}(\theta(u))=V_{u} P$, the vertical subspace of $T_{u} P$ for each point $u \in \mathcal{G}_{0}$, and $G_{0}$-equivariant, i.e. $\left(r^{g}\right)^{*} \theta=\operatorname{Ad}\left(g^{-1}\right) \circ \theta$ for all $g \in G_{0}$, where $r^{g}$ denotes the right principal action of $g$.

If $\mathfrak{g}$ is of projective type, then $G_{0} \cong G L\left(\mathfrak{g}_{-1}\right)$, so $\mathcal{G}_{0}$ is just the full frame bundle in this case. The whole structure is then given by the choice of an equivalence class of connections, see 4.4 below. 
As mentioned above, examples of irreducible parabolic geometries include the classical projective, conformal and almost Grassmannian (among them almost quaternionic) structures. See [8, part I, 3.3] for further examples and a more detailed discussion.

4.2. The first thing one gets from a structure $\left(\mathcal{G}_{0}, \theta\right)$ as described above is an identification of the tangent bundle $T M$ of $M$ with the associated bundle $\mathcal{G}_{0} \times{ }_{G_{0}} \mathfrak{g}_{-1}$ corresponding to the adjoint action of $G_{0}$ on $\mathfrak{g}_{-1}$. Explicitly, the isomorphism $\mathcal{G}_{0} \times{ }_{G_{0}} \mathfrak{g}_{-1} \rightarrow T M$ is induced by the map $\mathcal{G}_{0} \times \mathfrak{g}_{-1} \rightarrow T M$ given by mapping $(u, X)$ to $T_{u} p \cdot \xi$, where $\xi \in T_{u} \mathcal{G}_{0}$ is any element with $\theta(\xi)=X$. Since $\theta$ is strictly horizontal, this map is well defined and surjective on each fiber, and since $\theta$ is equivariant, it descends to the required isomorphism.

As we have observed in 2.8 , the choice of a $G$-invariant bilinear form $B$ on $\mathfrak{g}$ induces a non-degenerate, $G_{0}$-invariant pairing between $\mathfrak{g}_{-1}$ and $\mathfrak{g}_{1}$, so $\mathfrak{g}_{1}$ is the dual $G_{0}$-module to $\mathfrak{g}_{-1}$. In particular, this implies that the cotangent bundle $T^{*} M$ is isomorphic to the associated bundle $\mathcal{G}_{0} \times{ }_{G_{0}} \mathfrak{g}_{1}$.

We can also form the associated bundle $\mathcal{G}_{0} \times{ }_{G_{0}} \mathfrak{g}_{0}$. For reasons that will become clear soon, we denote this bundle by $\operatorname{End}_{0} T M$.

The Lie bracket on $\mathfrak{g}$ is equivariant under the adjoint action and so, in particular, it is equivariant under the action of $G_{0}$. Moreover, since the action of $G_{0}$ preserves the grading on $\mathfrak{g}$, we can split the bracket into components and get induced vector bundle homomorphisms on the associated bundles. Since using the Jacobi-identity to relate various brackets will be essential in the sequel, we will denote all these induced brackets by $\{$,$\} . In detail, the induced brackets annihilate T M \otimes T M$ and $T^{*} M \otimes T^{*} M$ and we have non-trivial brackets $T M \otimes \operatorname{End}_{0} T M \rightarrow T M, T M \otimes$ $T^{*} M \rightarrow \operatorname{End}_{0} T M, \operatorname{End}_{0} T M \otimes \operatorname{End}_{0} T M \rightarrow \operatorname{End}_{0} T M$, and $\operatorname{End}_{0} T M \otimes T^{*} M \rightarrow$ $T^{*} M$. These brackets are the key input for our further constructions.

It is well known that for all $|k|$-graded simple Lie algebras the map $\mathfrak{g}_{0} \rightarrow$ $L\left(\mathfrak{g}_{-1}, \mathfrak{g}_{-1}\right)$ induced by the bracket $\mathfrak{g}_{0} \times \mathfrak{g}_{-1} \rightarrow \mathfrak{g}_{-1}$ is injective (see e.g. [33, lemma $3.2]$ ). Moreover, $\mathfrak{g}_{0}$ is always reductive and in the $|1|$-graded case $\mathfrak{g}_{-1}$ is an irreducible $\mathfrak{g}_{0}$ module. In particular, this implies that via $\{$,$\} we can view \operatorname{End}_{0} T M$ as a subbundle of $L(T M, T M) \cong T^{*} M \otimes T M$. Note that if $\mathfrak{g}$ is of projective type, we simply have $\operatorname{End}_{0} T M=T^{*} M \otimes T M$. Note further, that under the identification of $\operatorname{End}_{0} T M$ with a subbundle of $T^{*} M \otimes T M$, the algebraic bracket on $\operatorname{End}_{0} T M$ is simply given by the commutator of endomorphisms.

Proposition 4.3. Let $\mathcal{G}_{0} \rightarrow M$ be an irreducible parabolic geometry. Let $x \in M$, $\left\{X_{i}\right\}$ be a basis for $T_{x} M$ and $\left\{Z_{i}\right\}$ the dual basis for $T_{x}^{*} M$.

(1) For each element $\Phi \in T_{x}^{*} M \otimes T_{x} M$ there is a unique element $A \in \operatorname{End}_{0} T_{x} M$ such that $\sum_{i}\left\{Z_{i}, \Phi\left(X_{i}\right)\right\}=\sum_{i}\left\{Z_{i},\left\{A, X_{i}\right\}\right\}$.

(2) For each element $\Phi \in T_{x}^{*} M \otimes \operatorname{End}_{0} T_{x} M$ there is a unique element $\omega \in T_{x}^{*} M$ such that $\sum_{i}\left\{Z_{i}, \Phi\left(X_{i}\right)\right\}=\sum_{i}\left\{Z_{i},\left\{\omega, X_{i}\right\}\right\}$. If $\mathfrak{g}$ is not of projective type, then there is an element $\tau \in \Lambda^{2} T^{*} M \otimes T M$ such that $\Phi(\xi)=\{\omega, \xi\}+\sum_{i}\left\{Z_{i}, \tau\left(\xi, X_{i}\right)\right\}$ for all $\xi \in T_{x} M$. Moreover, we can choose $\tau=0$, i.e. $\Phi(\xi)=\{\omega, \xi\}$ if and only if $\{\Phi(\xi), \eta\}=\{\Phi(\eta), \xi\}$ for all elements $\xi, \eta \in T_{x} M$.

(3) For each element $\tau \in \Lambda^{2} T_{x}^{*} M \otimes T_{x} M$ there is an element $\Phi \in T_{x}^{*} M \otimes$ End $_{0} T_{x} M$ such that $\sum_{i}\left\{Z_{i}, \tau\left(\xi, X_{i}\right)\right\}=\sum_{i}\left\{Z_{i},\left\{\Phi(\xi), X_{i}\right\}-\left\{\Phi\left(X_{i}\right), \xi\right\}\right\}$. Moreover, if $\mathfrak{g}$ is not of projective type and $\Phi_{1}, \Phi_{2}$ are two such elements, then there is a unique element $\omega \in T_{x}^{*} M$ such that $\Phi_{2}(\xi)=\Phi_{1}(\xi)+\{\omega, \xi\}$. 
(4) For each element $\kappa \in \Lambda^{2} T_{x}^{*} M \otimes \operatorname{End}_{0} T_{x} M$ there exists a unique element $\Psi \in L\left(T_{x} M, T_{x}^{*} M\right) \cong T_{x}^{*} M \otimes T_{x}^{*} M$ such that

$$
\sum_{i}\left\{Z_{i}, \kappa\left(\xi, X_{i}\right)\right\}=\sum_{i}\left\{Z_{i},\left\{\Psi(\xi), X_{i}\right\}-\left\{\Psi\left(X_{i}\right), \xi\right\}\right\} \quad \text { for all } \xi \in T_{x} M
$$

All elements, whose existence is asserted by these statements are independent of the choice of the basis $\left\{X_{i}\right\}$. Moreover, the analogous statements hold for smooth sections instead of elements at one point.

Proof. These statements can be proved for each structure separately by straightforward but tedious computations. There is, however, a uniform proof for all structures based on the analysis of the standard complex computing the Lie algebra cohomology $H^{*}\left(\mathfrak{g}_{-1}, \mathfrak{g}\right)$ and basic results on these cohomology groups: By definition, the $k$-th space $C^{k}\left(\mathfrak{g}_{-1}, \mathfrak{g}\right)$ in this standard complex is the space of all $k$-linear, alternating maps $\left(\mathfrak{g}^{-1}\right)^{k} \rightarrow \mathfrak{g}$, so $C^{k}\left(\mathfrak{g}_{-1}, \mathfrak{g}\right)=\Lambda^{k} g_{-1}^{*} \otimes \mathfrak{g}$. The differential $\partial: C^{k}\left(\mathfrak{g}_{-1}, \mathfrak{g}\right) \rightarrow$ $C^{k+1}\left(\mathfrak{g}_{-1}, \mathfrak{g}\right)$ is defined by $\partial(\varphi)\left(Y_{0}, \ldots, Y_{k}\right):=\sum_{i=0}^{k}(-1)^{i}\left[Y_{i}, \varphi\left(Y_{0}, \ldots, \hat{i}, \ldots, Y_{k}\right)\right]$. (The second term in the usual formula for the Lie algebra differential does not occur, since $\mathfrak{g}_{-1}$ is Abelian.)

Now let $\partial^{*}: C^{k+1}\left(\mathfrak{g}_{-1}, \mathfrak{g}\right) \rightarrow C^{k}\left(\mathfrak{g}_{-1}, \mathfrak{g}\right)$ be the codifferential from 2.10 which, as we observed there, is an adjoint to $\partial$. In the $|1|$-graded case, the formula for $\partial^{*}$ is given by $\partial^{*} \varphi\left(Y_{1}, \ldots, Y_{k}\right)=\sum_{i}\left[b_{i}, \varphi\left(a_{i}, Y_{1}, \ldots, Y_{k}\right)\right]$, where $\left\{a_{i}\right\}$ is a basis of $\mathfrak{g}_{-1}$ and $\left\{b_{i}\right\}$ is the dual basis of $\mathfrak{g}_{1}$.

Next, one defines the Laplacian $\square: C^{k}\left(\mathfrak{g}_{-1}, \mathfrak{g}\right) \rightarrow C^{k}\left(\mathfrak{g}_{-1}, \mathfrak{g}\right)$ by $\square=\partial \circ \partial^{*}+\partial^{*} \circ$ $\partial$. Since $\partial$ and $\partial^{*}$ are adjoint, and $\partial^{2}=\left(\partial^{*}\right)^{2}=0$, we get $\operatorname{ker}(\square)=\operatorname{ker}(\partial) \cap \operatorname{ker}\left(\partial^{*}\right)$ and a Hodge-decomposition $C^{k}\left(\mathfrak{g}_{-1}, \mathfrak{g}\right)=\operatorname{im}(\partial) \oplus \operatorname{ker}(\square) \oplus \operatorname{im}\left(\partial^{*}\right)$. Moreover, $\operatorname{ker}(\partial)=\operatorname{im}(\partial) \oplus \operatorname{ker}(\square)$ and similarly for $\partial^{*}$, so in particular, $\operatorname{ker}(\square) \cong H^{k}\left(\mathfrak{g}_{-1}, \mathfrak{g}\right)$.

Now there are a few things to observe: First of all, one easily verifies that $\partial$ is a $G_{0}$-homomorphisms and as we have noted in 2.10, $\partial^{*}$ is even a $P$-homomorphism. Hence also $\square$ is a $G_{0}$-homomorphism and the the Hodge decomposition is a decomposition of $G_{0}$-modules. Moreover, the splitting $\mathfrak{g}=\mathfrak{g}_{-1} \oplus \mathfrak{g}_{0} \oplus \mathfrak{g}_{1}$ of $G_{0}$-modules induces a splitting of $C^{k}\left(\mathfrak{g}_{-1}, \mathfrak{g}\right)$ into $G_{0}$ submodules and this splitting is given by homogeneous degrees of multilinear maps. Now obviously both $\partial$ and $\partial^{*}$ preserve homogeneous degrees, so also the Hodge decomposition induces a decomposition for each homogeneous degree.

Notice that the projections describing the Hodge-decomposition can be explicitly described in terms of $\partial, \partial^{*}$ and $\square$. By construction, $\operatorname{im}(\square) \subset \operatorname{im}(\partial) \oplus \operatorname{im}\left(\partial^{*}\right)$, so in particular $\operatorname{ker}(\square) \cap \operatorname{im}(\square)=\{0\}$. From this, one immediately verifies that $\square$ restricts to invertible $G_{0}$-homomorphisms $\operatorname{im}(\partial) \rightarrow \operatorname{im}(\partial)$ and $\operatorname{im}\left(\partial^{*}\right) \rightarrow \operatorname{im}\left(\partial^{*}\right)$, so it makes sense to form $\square^{-1} \circ \partial$ and $\square^{-1} \circ \partial^{*}$, even though $\square$ is not invertible in general. It is then simple to verify that the projections from $C^{k}\left(\mathfrak{g}_{-1}, \mathfrak{g}\right)$ to $\operatorname{im}(\partial)$ and $\operatorname{im}\left(\partial^{*}\right)$ are given by $\partial \circ \square^{-1} \circ \partial^{*}$ and $\partial^{*} \circ \square^{-1} \circ \partial$, respectively.

Finally we need some facts about the cohomology $H^{*}\left(\mathfrak{g}_{-1}, \mathfrak{g}\right)$ provided by Kostant's version of the Bott-Borel-Weil theorem. Namely, for any $|1|$-graded Lie algebra $\mathfrak{g}$ the cohomology in degree zero is given by $H^{0}\left(\mathfrak{g}_{-1}, \mathfrak{g}\right)=\mathfrak{g}_{-1}$ and if $\mathfrak{g}$ is not of projective type, then the first cohomology $H^{1}\left(\mathfrak{g}_{-1}, \mathfrak{g}\right)$ is contained in $\mathfrak{g}_{-1}^{*} \otimes \mathfrak{g}_{-1} \subset \mathfrak{g}_{-1}^{*} \otimes \mathfrak{g}$. If $\mathfrak{g}$ is of projective type, then $H^{1}\left(\mathfrak{g}_{-1}, \mathfrak{g}\right)$ is contained in $\mathfrak{g}_{-1}^{*} \otimes \mathfrak{g}_{0}$. See [28] for a proof of these results in the $|1|$-graded case. 
Now we only have to transfer these results to the manifold in order to obtain the proof of our proposition. Since all maps we are dealing with are $G_{0^{-}}$ homomorphisms, they induce vector bundle homomorphisms between the corresponding associated bundles, and also the Hodge decomposition induces a decomposition of the bundles into smooth subbundles. Then (1) is exactly the decomposition $\mathfrak{g}_{-1}^{*} \otimes \mathfrak{g}_{1}=\operatorname{im}(\partial) \oplus \operatorname{ker}\left(\partial^{*}\right)$ plus the fact that $\partial: \mathfrak{g}_{0} \rightarrow \mathfrak{g}_{-1}^{*} \otimes \mathfrak{g}_{-1}$ is injective since $H^{0}\left(\mathfrak{g}_{-1}, \mathfrak{g}\right) \cap \mathfrak{g}_{0}=0$.

The first part of (2) is the decomposition $\mathfrak{g}_{-1}^{*} \otimes \mathfrak{g}_{0}=\operatorname{im}(\partial) \oplus \operatorname{ker}\left(\partial^{*}\right)$ plus the fact that $\partial: \mathfrak{g}_{1} \rightarrow \mathfrak{g}_{-1}^{*} \otimes \mathfrak{g}_{0}$ is injective. If $\mathfrak{g}$ is not of projective type, then $H^{1}\left(\mathfrak{g}_{-1}, \mathfrak{g}\right) \subset \mathfrak{g}_{-1}^{*} \otimes \mathfrak{g}_{-1}$, so the Hodge decomposition of $\mathfrak{g}_{-1}^{*} \otimes \mathfrak{g}_{0}$ just reads as $\operatorname{im}(\partial) \oplus \operatorname{im}\left(\partial^{*}\right)$ and this immediately gives the second part of (2). For the very last comment of that part note that if $\tau=0$, then the claimed symmetry follows from the Jacobi identity and the triviality of the bracket on $T M \otimes T M$. On the other hand if $\{\Phi(\xi), \eta\} \neq\{\Phi(\eta), \xi\}$, for some $\xi, \eta \in T_{x} M$, then $\Phi$ corresponds to an element of $\mathfrak{g}_{-1}^{*} \otimes \mathfrak{g}_{0}$ outside $\operatorname{ker}(\partial)$ and thus $\partial \partial^{*}$ does not annihilate the element of $\Lambda^{2} \mathfrak{g}_{-1}^{*} \otimes \mathfrak{g}_{-1}$ corresponding to $\tau$.

Statement (3) reflects the decomposition $\Lambda^{2} \mathfrak{g}_{-1}^{*} \otimes \mathfrak{g}_{-1}=\operatorname{im}(\partial) \oplus \operatorname{ker}\left(\partial^{*}\right)$, and the fact that if $\mathfrak{g}$ is not of projective type, then the kernel of $\partial: \mathfrak{g}_{-1}^{*} \otimes \mathfrak{g}_{0} \rightarrow \Lambda^{2} \mathfrak{g}_{-1}^{*} \otimes \mathfrak{g}_{-1}$ coincides with the image of $\partial: \mathfrak{g}_{1} \rightarrow \mathfrak{g}_{-1}^{*} \otimes \mathfrak{g}_{0}$, since $H^{1}\left(\mathfrak{g}_{-1}, \mathfrak{g}\right)$ is contained in $\mathfrak{g}_{-1}^{*} \otimes \mathfrak{g}_{-1}$.

The existence part of statement (4) follows from the Hodge decomposition $\Lambda^{2} \mathfrak{g}_{-1}^{*} \otimes \mathfrak{g}_{0}=\operatorname{im}(\partial) \oplus \operatorname{ker}\left(\partial^{*}\right)$. Since there is no map $\partial$ landing in $\mathfrak{g}_{-1}^{*} \otimes \mathfrak{g}_{1}$ by homogeneity, the fact that $H^{1}\left(\mathfrak{g}_{-1}, \mathfrak{g}\right) \cap \mathfrak{g}_{-1}^{*} \otimes \mathfrak{g}_{1}=\{0\}$ implies that $\partial: \mathfrak{g}_{-1}^{*} \otimes \mathfrak{g}_{1} \rightarrow$ $\Lambda^{2} \mathfrak{g}_{-1}^{*} \otimes \mathfrak{g}_{0}$ is injective, which gives the uniqueness part in statement (4).

The fact that all statements are independent of the choice of the basis for $T_{x} M$ immediately follows since all maps involving the bases are induced by $\partial^{*}$, which in turn is independent of the choice of the basis of $\mathfrak{g}_{-1}$. Finally, the fact that analogous results hold for smooth sections is clear from the explicit description of the projectors above.

4.4. Preferred connections. If $\mathfrak{g}$ is of projective type, then the irreducible parabolic geometry is precisely a class of affine connections on $M$ having $\partial^{*}$-closed torsion, such that for two connections $\nabla$ and $\hat{\nabla}$ in this class there is a (necessarily unique) smooth one-form $\Upsilon \in \Omega^{1}(M)$ such that $\hat{\nabla}_{\xi} \eta=\nabla_{\xi} \eta+\{\{\Upsilon, \xi\}, \eta\}$. (It is easy to see that then all the connections in the class in fact have the same torsion, see below.) For any other irreducible parabolic geometry we can use part (3) of proposition 4.3 to define a class of preferred affine connections on $M$ having analogous properties. Consider two affine connections $\nabla$ and $\hat{\nabla}$ on $M$, which both are induced by principal connections on $\mathcal{G}_{0}$. A difference of two affine connections, $\hat{\nabla}-\nabla$, is a section of $T^{*} M \otimes T^{*} M \otimes T M$. However, since they both are induced by principal connection on $\mathcal{G}_{0}$, the difference must actually be a smooth section of the subbundle $T^{*} M \otimes \operatorname{End}_{0} T M$. Conversely, if $\nabla$ is induced by a principal connection on $\mathcal{G}_{0}$ and $\Phi$ is an arbitrary smooth section of $T^{*} M \otimes \operatorname{End}_{0} T M$, then $\hat{\nabla}_{\xi} \eta=\nabla_{\xi} \eta+\{\Phi(\xi), \eta\}$ is also induced by a principal connection on $\mathcal{G}_{0}$. Moreover, if $T$ is the torsion of $\nabla$, then the torsion $\hat{T}$ of $\hat{\nabla}$ is given by $\hat{T}(\xi, \eta)=T(\xi, \eta)+\{\Phi(\xi), \eta\}-\{\Phi(\eta), \xi\}$. Consequently, if we start with any connection $\nabla$ we can find by proposition 4.3 3 ) a section $\Phi$ of $T^{*} M \otimes \operatorname{End}_{0} T M$ such that the corresponding connection $\hat{\nabla}$ has the 
property that $\sum_{i}\left\{Z_{i}, \hat{T}\left(\xi, X_{i}\right)\right\}=0$ for any vector field $\xi$ on $M$ and any local frame $\left\{X_{i}\right\}$ for $T M$ with dual frame $\left\{Z_{i}\right\}$ of $T^{*} M$.

Definition. If $\mathfrak{g}$ is not of projective type, then an affine connection $\nabla$ on $M$ is called a preferred connection for the irreducible parabolic geometry $\mathcal{G}_{0} \rightarrow M$ if and only if it is induced by a principal connection on $\mathcal{G}_{0}$ and its torsion $T$ has the property that $\sum_{i}\left\{Z_{i}, T\left(\xi, X_{i}\right)\right\}=0$ for any vector field $\xi$ on $M$ and any local frame $\left\{X_{i}\right\}$ for $T M$ with dual frame $\left\{Z_{i}\right\}$ of $T^{*} M$.

As we saw above, preferred connections exist. Moreover, if both $\nabla$ and $\hat{\nabla}$ are preferred connections on $M$, and $\Phi=\hat{\nabla}-\nabla \in \Gamma\left(T^{*} M \otimes \operatorname{End}_{0} T M\right)$, then since both connections are preferred, we must have $\sum_{i}\left\{Z_{i},\left\{\Phi(\xi), X_{i}\right\}-\left\{\Phi\left(X_{i}\right), \xi\right\}\right\}=0$. Again by proposition 4.3 (3) this implies that there is a unique one form $\Upsilon \in \Omega^{1}(M)$ such that $\Phi(\xi)=\{\Upsilon, \xi\}$. But by the Jacobi-identity, and since $\{\xi, \eta\}=0$, we see that $\{\{\Upsilon, \xi\}, \eta\}$ is symmetric in $\xi$ and $\eta$, so $\hat{T}=T$. Consequently, all preferred connections have the same torsion and so this is an invariant of the parabolic geometry, which is called the torsion $T$ of $M$.

Whether this torsion may actually be nonzero depends on the concrete choice of the Lie algebra $\mathfrak{g}$. The point about this is that the Lie algebra differential $\partial$ is identically zero on $\Lambda^{2} \mathfrak{g}_{-1}^{*} \otimes \mathfrak{g}_{-1}$ and so, since the torsion takes values in $\operatorname{ker}\left(\partial^{*}\right)=$ $\operatorname{ker}(\square)$, it can only be nonzero if there is a component of the cohomology $H^{2}\left(\mathfrak{g}_{-1}, \mathfrak{g}\right)$ contained in that subspace. Again, this cohomology group can be computed using Kostant's version of the Bott-Borel-Weil theorem for each choice of $\mathfrak{g}$. For example, for conformal structures the cohomology is contained in $\Lambda^{2} \mathfrak{g}_{-1}^{*} \otimes \mathfrak{g}_{0}$ in dimensions bigger than three and in $\Lambda^{2} \mathfrak{g}_{-1}^{*} \otimes \mathfrak{g}_{1}$ in dimension three. In particular, the torsion is always zero for conformal structures. On the other hand, there are also irreducible parabolic geometries for which the torsion is a complete obstruction against local flatness. See [7] for a discussion of the use of second cohomology in describing obstructions against local flatness for irreducible parabolic geometries.

4.5. For later use, we have to analyze the set of preferred connections more closely. In particular, we discuss the difference between the curvatures of different preferred connections. Moreover, the formulae we derive are useful for making concrete computations in any specific structure.

From 4.4 we know that for any irreducible parabolic geometry we have a class of preferred affine connections on $M$ and for two such connections $\nabla$ and $\hat{\nabla}$ there is a unique one-form $\Upsilon \in \Omega^{1}(M)$ such that $\hat{\nabla}_{\xi} \eta=\nabla_{\xi} \eta+\{\{\Upsilon, \xi\}, \eta\}$. Using this, one immediately verifies that for a one-form $\omega \in \Omega^{1}(M)$ we get $\hat{\nabla}_{\xi} \omega=$ $\nabla_{\xi} \omega+\{\{\Upsilon, \xi\}, \omega\}$. From this in turn, one verifies that for a section $\Phi$ of $L(T M, T M)$ the transformation is given by $\hat{\nabla}_{\xi} \Phi=\nabla_{\xi} \Phi+\{\Upsilon, \xi\} \circ \Phi-\Phi \circ\{\Upsilon, \xi\}$. In particular, if $\Phi$ is a section of the subbundle $\operatorname{End}_{0} T M$, then $\hat{\nabla}_{\xi} \Phi=\nabla_{\xi} \Phi+\{\{\Upsilon, \xi\}, \Phi\}$.

To compute the formulae for second derivatives, we have to keep in mind that the brackets $\{$,$\} are defined on bundles induced from \mathcal{G}_{0}$ by $G_{0}$-homomorphisms of the corresponding modules. Thus, they are covariantly constant for any affine connection on $M$ which is induced by a principal connection on $\mathcal{G}_{0}$ and hence, in particular, for all preferred connections. Thus we compute

$$
\hat{\nabla}_{\xi}(\hat{\nabla} \eta)=\hat{\nabla}_{\xi}(\nabla \eta+\{\Upsilon, \eta\})=\hat{\nabla}_{\xi}(\nabla \eta)+\hat{\nabla}_{\xi}(\{\Upsilon, \eta\})
$$

and, since $\{\Upsilon, \eta\}$ is a section of $\operatorname{End}_{0} T M$, we end up with

$$
\nabla_{\xi}(\nabla \eta)+\{\Upsilon, \xi\} \circ \nabla \eta-\nabla \eta \circ\{\Upsilon, \xi\}+\left\{\nabla_{\xi} \Upsilon, \eta\right\}+\left\{\Upsilon, \nabla_{\xi} \eta\right\}+\{\{\Upsilon, \xi\},\{\Upsilon, \eta\}\} .
$$


Using the results on second derivatives, we can next compute the change of curvature. From the definition of the curvature $R$ of an affine connection $\nabla$ one immediately concludes that this can be written as

$$
R(\xi, \eta)(\zeta)=\nabla_{\xi}(\nabla \zeta)(\eta)-\nabla_{\eta}(\nabla \zeta)(\xi)+\nabla_{T(\xi, \eta)} \zeta,
$$

where $T$ denotes the torsion of $\nabla$. Inserting into the formula above, we get

$$
\begin{aligned}
& \hat{\nabla}_{\xi}(\hat{\nabla} \zeta)(\eta)=\nabla_{\xi}(\nabla \zeta)(\eta)+\left\{\{\Upsilon, \xi\}, \nabla_{\eta} \zeta\right\}-\nabla_{\{\{\Upsilon, \xi\}, \eta\}} \zeta \\
& \quad+\left\{\left\{\nabla_{\xi} \Upsilon, \zeta\right\}, \eta\right\}+\left\{\left\{\Upsilon, \nabla_{\xi} \zeta\right\}, \eta\right\}+\{\{\{\Upsilon, \xi\},\{\Upsilon, \zeta\}\}, \eta\} .
\end{aligned}
$$

In the second term of the right-hand side, we may exchange $\xi$ and $\nabla_{\eta} \zeta$ and so this term and the fifth one cancel after alternation in $\xi$ and $\eta$. Moreover, the third term in the right-hand side is symmetric in $\xi$ and $\eta$, so it also vanishes after alternation. Finally a short computation using the Jacobi identity shows that after alternation the last term just produces $\{\{\{\Upsilon, \xi\},\{\Upsilon, \eta\}\}, \zeta\}$. Bringing $\zeta$ to the right in the fourth term we arrive at

$$
\hat{R}(\xi, \eta)=R(\xi, \eta)+\left\{\nabla_{\xi} \Upsilon, \eta\right\}-\left\{\nabla_{\eta} \Upsilon, \xi\right\}+\{\{\Upsilon, \xi\},\{\Upsilon, \eta\}\}+\{\Upsilon, T(\xi, \eta)\} .
$$

4.6. The Rho-tensor, Weyl-curvature and the Cotton-York tensor. We can split the curvature of a preferred connection into parts that have simpler transformation laws than the curvature itself. Since any preferred connection $\nabla$ is induced from a principal connection on $\mathcal{G}_{0}$, its curvature $R$ is a section of $\Lambda^{2} T^{*} \otimes$ End $_{0} T M$. Hence, by proposition 4.3(4), there is a unique section $\mathrm{P}$ of the bundle $L\left(T M, T^{*} M\right) \cong T^{*} M \otimes T^{*} M$ such that $W(\xi, \eta):=R(\xi, \eta)+\{\mathrm{P}(\xi), \eta\}-\{\mathrm{P}(\eta), \xi\}$ has the property that $\sum_{i}\left\{Z_{i}, W\left(\xi, X_{i}\right)\right\}=0$ for all vector fields $\xi$ on $M$ and one (or equivalently any) local frame $\left\{X_{i}\right\}$ for $T M$ with dual frame $\left\{Z_{i}\right\}$. P is called the Rho-tensor of the preferred connection $\nabla$ and $W$ is called the Weyl-curvature of $\nabla$. Note that in abstract index notation $\sum_{i}\left\{Z_{i}, W\left(-, X_{i}\right)\right\}$ just equals $W_{a c b}{ }^{c}$. Using this, one easily verifies that for conformal structures, one recovers the usual Rho-tensor and Weyl-curvature.

Now observe that for all one-forms $\Upsilon$ and vector fields $\xi$, we have

$$
\sum_{i}\left\{Z_{i},\left\{\Upsilon, T\left(\xi, X_{i}\right)\right\}\right\}=\sum_{i}\left\{\Upsilon,\left\{Z_{i}, T\left(\xi, X_{i}\right)\right\}\right\}=0
$$

by construction of the torsion, see 4.4. Moreover, using the Jacobi identity, one easily verifies that

$$
-\frac{1}{2}(\{\{\Upsilon,\{\Upsilon, \xi\}\}, \eta\}-\{\{\Upsilon,\{\Upsilon, \eta\}\}, \xi\})=\{\{\Upsilon, \xi\},\{\Upsilon, \eta\}\} .
$$

Using these two facts and the formula for $\hat{R}(\xi, \eta)$ from 4.5, one easily verifies that if $\mathrm{P}$ is the Rho-tensor for $\nabla$ and $\hat{\nabla}$ is another preferred connection corresponding to $\Upsilon \in \Omega^{1}(M)$, then

$$
\hat{\mathrm{P}}(\xi)=\mathrm{P}(\xi)-\nabla_{\xi} \Upsilon+\frac{1}{2}\{\Upsilon,\{\Upsilon, \xi\}\}
$$

satisfies the defining equation for the Rho-tensor of $\hat{\nabla}$. Hence the uniqueness part of proposition 4.3(4) implies that this is the transformation law for the Rho-tensor. From this in turn, it follows that the Weyl-curvature of $\hat{\nabla}$ is given by

$$
\hat{W}(\xi, \eta)=W(\xi, \eta)+\{\Upsilon, T(\xi, \eta)\} .
$$

In particular, if the torsion of $M$ vanishes, then the Weyl-curvature is independent of the choice of the preferred connection, and thus an invariant of $M$. This always happens for conformal structures, see 4.4 
Recall that whether the torsion of a particular structure can possibly be nonzero depends on $H^{2}\left(\mathfrak{g}_{-}, \mathfrak{g}\right)$. Similarly, whether the Weyl curvature may be nonzero if the torsion vanishes, again depends on the cohomology $H^{2}\left(\mathfrak{g}_{-}, \mathfrak{g}\right)$. By $\underline{6}$, corollary $4.10]$ if the torsion vanishes, then the Weyl-curvature is not only $\partial^{*}$-closed but also $\partial$-closed, so zero torsion and nonzero Weyl-curvature may occur only if that cohomology group has a nonzero component contained in $\Lambda^{2} \mathfrak{g}_{-1}^{*} \otimes \mathfrak{g}_{0}$. For example, for conformal structures, the Weyl curvature is always zero in dimension three, while in higher dimensions it is a complete obstruction against local conformal flatness.

Finally, we define the Cotton-York tensor $C Y \in \Omega^{2}\left(M, T^{*} M\right)$ of a preferred connection $\nabla$ as the covariant exterior derivative of the Rho-tensor, which we may view as a $T^{*} M$-valued one-form. That is, by definition

$$
C Y(\xi, \eta)=\nabla_{\xi}(\mathrm{P}(\eta))-\nabla_{\eta}(\mathrm{P}(\xi))-\mathrm{P}([\xi, \eta]) .
$$

Inserting the definition of the torsion, we see that this can also be written as $(\nabla \mathrm{P})(\xi, \eta)-(\nabla \mathrm{P})(\eta, \xi)+\mathrm{P}(T(\xi, \eta))$. At this stage, one may verify by a direct computation that changing from $\nabla$ to $\hat{\nabla}$ the Cotton-York tensor transforms as follows:

$$
\widehat{C Y}(\xi, \eta)=C Y(\xi, \eta)+\{\Upsilon, W(\xi, \eta)\}+\frac{1}{2}\{\Upsilon,\{\Upsilon, T(\xi, \eta)\}\} .
$$

However, we will not need this, and it will drop out of the later developments automatically.

4.7. Now we can start the construction of the adjoint tractor bundle for the cases where $\mathfrak{g}$ is not of projective type. The first thing to observe is that there is an obvious invariant differential operator $d_{0}$ on vector fields on $M$ defined as follows: Let $\mathcal{F}$ be the quotient bundle $\left(T^{*} M \otimes T M\right) / \operatorname{End}_{0} T M$ and $\pi_{\mathcal{F}}: T^{*} M \otimes T M \rightarrow \mathcal{F}$ the canonical projection. Now for a preferred connection $\nabla$ on $M$ and a vector field $\xi \in \mathfrak{X}(M)$ consider $\pi_{\mathcal{F}}(\nabla \xi) \in \Gamma(\mathcal{F})$. If $\hat{\nabla}$ is another preferred connection, then by 4.4 there is a unique one-form $\Upsilon \in \Omega^{1}(M)$ such that $\hat{\nabla}_{\eta} \xi=\nabla_{\eta} \xi+\{\{\Upsilon, \eta\}, \xi\}$. Since the last expression is symmetric in $\xi$ and $\eta$, we can rewrite this equation as $\hat{\nabla} \xi=\nabla \xi+\{\Upsilon, \xi\}$. Since $\{\Upsilon, \xi\}$ is a section of $\operatorname{End}_{0} T M$, this implies that $\pi_{\mathcal{F}}(\hat{\nabla} \xi)=\pi_{\mathcal{F}}(\nabla \xi)$, so we obtain an invariant operator $d_{0}: \mathfrak{X}(M) \rightarrow \Gamma(\mathcal{F})$.

Next let $J^{1}(T M)$ be the first jet-prolongation of the tangent bundle of $M$. Then $d_{0}$ is induced by a vector bundle homomorphism $J^{1}(T M) \rightarrow \mathcal{F}$, which we also denote by $d_{0}$. From the construction it is clear that this bundle map is surjective, and we define $\mathcal{A}^{(0)}$ to be its kernel. Hence, by definition, we get a short exact sequence of vector bundles

$$
0 \rightarrow \mathcal{A}^{(0)} \longrightarrow J^{1}(T M) \stackrel{d_{0}}{\longrightarrow} \mathcal{F} \rightarrow 0 .
$$

Moreover, the jet exact sequence $0 \rightarrow T^{*} M \otimes T M \rightarrow J^{1}(T M) \rightarrow T M \rightarrow 0$ and the definition of $d_{0}$ immediately imply that we also get a short exact sequence

$$
0 \rightarrow \operatorname{End}_{0} T M \rightarrow \mathcal{A}^{(0)} \stackrel{p_{-1}}{\longrightarrow} T M \rightarrow 0 .
$$

Explicitly, the fiber $\mathcal{A}_{x}^{(0)}$ of $\mathcal{A}^{(0)}$ in $x \in M$ consists of all one-jets $j_{x}^{1}(\xi)$ at $x$ of vector fields $\xi$ such that for one (equivalently any) preferred connection $\nabla$ we have $\nabla \xi(x) \in \operatorname{End}_{0} T_{x} M$, and $p_{-1}\left(j_{x}^{1}(\xi)\right)=\xi(x)$.

Next, consider the first jet prolongation $J^{1}\left(d_{0}\right): J^{1}\left(J^{1}(T M)\right) \rightarrow J^{1}(\mathcal{F})$ of the bundle map $d_{0}$. Since forming a jet prolongation is an exact functor, $J^{1}\left(d_{0}\right)$ is surjective and its kernel is $J^{1}\left(\mathcal{A}^{(0)}\right)$. On the other hand, we have the subbundle 
$J^{2}(T M) \subset J^{1}\left(J^{1}(T M)\right)$, and we define $\mathcal{A} \subset J^{2}(T M)$ to be the kernel of the restriction of $J^{1}\left(d_{0}\right)$ to this subbundle. Equivalently, $\mathcal{A}=J^{1}\left(\mathcal{A}^{(0)}\right) \cap J^{2}(T M)$. By construction, we have a canonical projection $p_{0}: \mathcal{A} \rightarrow \mathcal{A}^{(0)}$, induced by the projection $J^{1}\left(\mathcal{A}^{(0)}\right) \rightarrow \mathcal{A}^{(0)}$.

Next, we have to analyze the kernel $\mathcal{K}$ of $p_{0}$. Clearly, $\mathcal{K}$ is the intersection of the kernel of the projection $J^{2}(T M) \rightarrow J^{1}(T M)$, which is just $S^{2} T^{*} M \otimes T M$, with the kernel of the projection $J^{1}\left(\mathcal{A}^{(0)}\right) \rightarrow \mathcal{A}^{(0)}$, which is $T^{*} M \otimes \mathcal{A}^{(0)}$. Thus, we get $\mathcal{K}=\left(T^{*} M \otimes \operatorname{End}_{0} T M\right) \cap\left(S^{2} T^{*} M \otimes T M\right)$. For a point $x \in M$ and an element $\Phi \in T_{x}^{*} M \otimes \operatorname{End}_{0} T_{x} M$, lying in $S^{2} T_{x}^{*} M \otimes T_{x} M$ just means $\{\Phi(\xi), \eta\}=\{\Phi(\eta), \xi\}$ for all $\xi, \eta \in T_{x} M$. But by proposition 4.3(2) this implies that there is a unique element $\omega \in T_{x}^{*} M$ such that $\Phi(\xi)=\{\omega, \xi\}$, which shows that $\mathcal{K}$ is canonically isomorphic to $T^{*} M$. In particular, this implies that $\mathcal{A}$ is a smooth subbundle of $J^{2}(T M)$, and we will prove that $\mathcal{A}$ is the normal adjoint tractor bundle on $M$.

Note that this result is the analogue in our picture of the fact that for a $|1|-$ graded Lie algebra $\mathfrak{g}$ which is not of projective type, the first prolongation of $\mathfrak{g}_{0} \subset$ $L\left(\mathfrak{g}_{-1}, \mathfrak{g}_{-1}\right)$ is exactly $\mathfrak{g}_{1}$.

We next define a filtration $\mathcal{A} \supset \mathcal{A}^{0} \supset \mathcal{A}^{1}$ on $\mathcal{A}$ by $\mathcal{A}^{0}=\operatorname{ker}\left(p_{-1} \circ p_{0}\right)$ and $\mathcal{A}^{1}=\mathcal{K}=\operatorname{ker}\left(p_{0}\right)$, where $p_{0}: \mathcal{A} \rightarrow \mathcal{A}^{(0)}$ and $p_{-1}: \mathcal{A}^{(0)} \rightarrow T M$ are the canonical projections. From above we know that $\mathcal{A}^{1} \cong T^{*} M$, while by construction $\mathcal{A} / \mathcal{A}^{0} \cong$ $T M$ and $\mathcal{A} / \mathcal{A}^{1} \cong \mathcal{A}^{(0)}$. In particular, this implies that $\mathcal{A}^{0} / \mathcal{A}^{1}$ is isomorphic to the kernel of $p_{-1}: \mathcal{A}^{(0)} \rightarrow T M$ and hence to $\operatorname{End}_{0} T M$. If we start with an atlas for $T M$ and use the induced atlas for $J^{2}(T M)$, then we see that $\mathcal{A}$ is a filtered vector bundle modelled on the filtered vector space $\mathfrak{g} \supset\left(\mathfrak{g}_{0} \oplus \mathfrak{g}_{1}\right) \supset \mathfrak{g}_{1}$, and thus is a candidate for an adjoint tractor bundle.

4.8. Next, we want to show that a choice of a preferred connection leads to an isomorphism $\mathcal{A} \cong T M \oplus \operatorname{End}_{0} T M \oplus T^{*} M$ of vector bundles, and compute how this isomorphism depends on the choice of the connection. In fact, several isomorphisms of that type are possible; the one that is described here has a particularly simple behavior under a change of preferred connection.

Let $\nabla$ be a preferred connection on $M$ and let $j_{x}^{2}(\xi)$ be an element of $\mathcal{A}_{x}$. Then we can form $\xi(x) \in T_{x} M$ and since $j_{x}^{2}(\xi) \in \mathcal{A}_{x}$, we know that $\nabla \xi(x) \in \operatorname{End}_{0} T_{x} M$, so the first two components of the isomorphism are very simple to describe. The third component is a little more complicated to get. Since $j_{x}^{2}(\xi) \in \mathcal{A}_{x}$, we know that $\nabla^{2} \xi(x)$ lies in $T_{x}^{*} M \otimes \operatorname{End}_{0} T_{x} M \subset T_{x}^{*} M \otimes T_{x}^{*} M \otimes T_{x} M$, so $\nabla_{\eta}(\nabla \xi)(x) \in \operatorname{End}_{0}(T M)$ for all $\eta$. Applying proposition $4.3(2)$ to the element of $T_{x}^{*} M \otimes \operatorname{End}_{0}(T M)$ giving the map

$$
\eta \mapsto \nabla_{\eta}(\nabla \xi)(x)+\{\mathrm{P}(\eta), \xi(x)\}
$$

gives us a unique element $\omega \in T_{x}^{*} M$ such that the map

$$
A(\eta):=\nabla_{\eta}(\nabla \xi)(x)+\{\mathrm{P}(\eta), \xi(x)\}-\{\omega, \eta\}
$$

has the property that $\sum_{i}\left\{Z_{i}, A\left(X_{i}\right)\right\}=0$ for one (or equivalently any) basis $\left\{X_{i}\right\}$ for $T_{x} M$ with dual basis $\left\{Z_{i}\right\}$ of $T_{x}^{*} M$.

Proposition. Choosing a preferred connection $\nabla$ on $M$, the map $j_{x}^{2}(\xi) \mapsto(\xi, \Phi, \omega)$, where $\xi=\xi(x), \Phi=\nabla \xi(x)$ and $\omega \in T_{x}^{*} M$ is defined as above, induces an isomorphism $\mathcal{A} \cong T M \oplus \operatorname{End}_{0} T M \oplus T^{*} M$ of vector bundles. If $\hat{\nabla}$ is another preferred connection corresponding to $\Upsilon \in \Omega^{1}(M)$, then the isomorphism corresponding to $\hat{\nabla}$ 
is given by

$$
(\widehat{\xi, \Phi, \omega})=\left(\xi, \Phi+\{\Upsilon, \xi\}, \omega+\{\Upsilon, \Phi\}+\frac{1}{2}\{\Upsilon,\{\Upsilon, \xi\}\}\right)
$$

Proof. The first two components of the map are clearly given by bundle maps, while for the last component this follows from the proof of proposition 4.3 Moreover, from 4.7 we know that the bundles $\mathcal{A}$ and $T M \oplus \operatorname{End}_{0} T M \oplus T^{*} M$ have the same rank, so to prove that we have constructed an isomorphism of bundles it suffices to show that the map we have constructed at a point is injective.

So let us assume that $j_{x}^{2}(\xi) \in \mathcal{A}_{x}$ is mapped to zero. Then vanishing of the first two components implies that $\xi(x)=0$ and $\nabla \xi(x)=0$, while by the proof of proposition 4.3 (2) the vanishing of the last component implies that we can recover $\nabla(\nabla \xi)(x)$ from its alternation. (Note that the term involving $\mathrm{P}$ does not show up, since $\xi(x)=0$.) But this alternation can be written in terms of $\xi(x)$ and $\nabla \xi(x)$ using the curvature and torsion of $\nabla$, so it has to be zero, too. Consequently, we must have $j_{x}^{2}(\xi)=0$, and we get an isomorphism of vector bundles.

If we change from $\nabla$ to $\hat{\nabla}$, then clearly $\xi(x)$ is independent of $\nabla$, while $\hat{\nabla} \xi=$ $\nabla \xi+\{\Upsilon, \xi\}$, so the transformation law for the first two components is obvious. For the last component, note that since $\nabla \xi(x) \in \operatorname{End}_{0} T_{x} M, \nabla_{\eta} \xi(x)=\{\nabla \xi(x), \eta\}$ and

$$
\{\{\Upsilon(x), \eta\}, \nabla \xi(x)\}+\{\Upsilon(x),\{\nabla \xi(x), \eta\}\}=\{\{\Upsilon(x), \nabla \xi(x)\}, \eta\},
$$

we see from 4.5 that at the point $x$ we get

$$
\hat{\nabla}_{\eta}(\hat{\nabla} \xi)=\nabla_{\eta}(\nabla \xi)+\{\{\Upsilon, \nabla \xi\}, \eta\}+\left\{\nabla_{\eta} \Upsilon, \xi\right\}+\{\{\Upsilon, \eta\},\{\Upsilon, \xi\}\}
$$

for all $\eta \in T_{x} M$. On the other hand, from 4.6 we know that

$$
\hat{\mathrm{P}}(\eta)=\mathrm{P}(\eta)-\nabla_{\eta} \Upsilon+\frac{1}{2}\{\Upsilon,\{\Upsilon, \eta\}\}
$$

Using these two facts, and the formula (1) from 4.6 one immediately sees that

$$
\omega+\{\Upsilon(x), \nabla \xi(x)\}+\frac{1}{2}\{\Upsilon(x),\{\Upsilon(x), \xi(x)\}\}
$$

satisfies the defining equation for the last component. Using the uniqueness part of proposition 4.3 (2) the transformation law follows.

Remark. The proof of proposition 4.3 gives us a simple way to compute the last component $\omega$ in our isomorphism explicitly. Namely, we see that we can obtain $\omega$ by applying $\square^{-1} \circ \partial^{*}$ to our given element of $T_{x}^{*} M \otimes \operatorname{End}_{0} T_{x} M$. But since $T^{*} M$ corresponds to the irreducible representation $\mathfrak{g}_{1}$ of $G_{0}$, the Laplacian on that space is just a scalar multiple of the identity. Hence, if we denote by $\lambda$ the inverse of this scalar (which depends only on $\mathfrak{g}$ ), then

$$
\omega=\lambda\left(\sum_{i}\left\{Z_{i}, \nabla_{X_{i}}(\nabla \xi)(x)+\left\{\mathrm{P}\left(X_{i}\right), \xi(x)\right\}\right\}\right) .
$$

4.9. The adjoint tractor bundle for $\mathfrak{g}$ of projective type. For $\mathfrak{g}$ of projective type, there is a simple direct construction of the adjoint tractor bundle as follows: Consider the two jet prolongations $J^{2}(T M)$ of the tangent bundle of $M$. Then this contains the subbundle $S^{2} T^{*} M \otimes T M$ as the kernel of the canonical projection to $J^{1}(T M)$. Now there is a unique trace $S^{2} T^{*} M \otimes T M \rightarrow T^{*} M$, and we denote its kernel by $\left(S^{2} T^{*} M \otimes T M\right)_{0}$. This is also a smooth subbundle of $J^{2}(T M)$ and we define $\mathcal{A}$ to be the quotient bundle $J^{2}(T M) /\left(S^{2} T^{*} M \otimes T M\right)_{0}$. Note that on one hand this implies that the rank of $\mathcal{A}$ equals the rank of $T M \oplus\left(T^{*} M \otimes T M\right) \oplus T^{*} M$, and on the other hand it immediately gives a canonical second order differential 
operator from vector fields on $M$ to sections of $\mathcal{A}$ by composing the canonical projection $J^{2}(T M) \rightarrow \mathcal{A}$ with the two-jet operator.

Now we can also easily get the analogue of proposition 4.8 for $\mathfrak{g}$ of projective type. Choosing a preferred connection $\nabla$, we can define a bundle map $J^{2}(T M) \rightarrow$ $T M \oplus T^{*} M \otimes T M \oplus T^{*} M$ by mapping $j_{x}^{2}(\xi)$ to $(\xi(x), \nabla \xi(x), \omega)$, where $\omega$ is defined as in 4.8 above. (Recall that $\operatorname{End}_{0}(T M)=T^{*} M \otimes T M$ for $\mathfrak{g}$ of projective type.) If $j_{x}^{2}(\xi)$ goes to zero under this mapping, then clearly $j_{x}^{1}(\xi)=0$ and so, in particular, $\nabla^{2} \xi(x)$ is symmetric. Hence, by proposition $4.3(2), \omega=0$, in this case, if and only if $j_{x}^{2}(\xi)$ lies in $\left(S^{2} T_{x}^{*} M \otimes T_{x} M\right)_{0}$. Consequently, this bundle map factors to an injection $\mathcal{A} \rightarrow T M \oplus T^{*} M \otimes T M \oplus T^{*} M$, which must be an isomorphism since both bundles have the same rank. Finally, the change of this isomorphism under a change of the preferred connection is computed exactly as in the proof of proposition 4.8 Thus, proposition 4.8 also holds for $\mathfrak{g}$ of projective type.

4.10. The algebraic bracket. Via the isomorphism $\mathcal{A} \cong T M \oplus \operatorname{End}_{0} T M \oplus T^{*} M$ induced by the choice of a preferred connection, it is now very simple to construct the algebraic bracket on $\mathcal{A}$. Namely, choose a preferred connection $\nabla$ on $M$, use it to identify $\mathcal{A}$ with $T M \oplus \operatorname{End}_{0} T M \oplus T^{*} M$ and define the algebraic bracket $\{$, on $\mathcal{A}$ by

$$
\begin{aligned}
& \{(\xi, \Phi, \omega),(\eta, \Psi, \tau)\} \\
& \quad:=(\{\xi, \Psi\}+\{\Phi, \eta\},\{\Phi, \Psi\}+\{\xi, \tau\}+\{\omega, \eta\},\{\Phi, \tau\}+\{\omega, \Psi\}) .
\end{aligned}
$$

Proposition. The definition of the algebraic bracket above is independent of the choice of the preferred connection $\nabla .(\mathcal{A},\{\}$,$) is a bundle of filtered Lie-algebras$ modelled on the filtered Lie algebra $\mathfrak{g} \supset \mathfrak{g}_{0} \oplus \mathfrak{g}_{1} \supset \mathfrak{g}_{1}$.

Proof. Recall that $T M, \operatorname{End}_{0} T M$, and $T^{*} M$ are associated to $\mathcal{G}_{0}$, corresponding to the representations $\mathfrak{g}_{-1}, \mathfrak{g}_{0}$, and $\mathfrak{g}_{1}$, respectively. Hence, the bracket we put on the triples just means that we declare the isomorphisms $T_{x} M \oplus \operatorname{End}_{0} T_{x} M \oplus T_{x}^{*} M \cong \mathfrak{g}$ given by the points $u \in\left(\mathcal{G}_{0}\right)_{x}$ to be isomorphisms of graded Lie-algebras, and then pull back this bracket to $\mathcal{A}$ via the isomorphism given by the choice of a preferred connection. If we change to another connection $\hat{\nabla}$ corresponding to $\Upsilon \in$ $\Omega^{1}(M)$, then for each point $u \in\left(\mathcal{G}_{0}\right)_{x}$, the value $\Upsilon(x)$ corresponds to an element $Z \in \mathfrak{g}_{1}$. But then by proposition 4.8 the change in the identifications of $\mathcal{A}_{x}$ with $T_{x} M \oplus \operatorname{End}_{0} T_{x} M \oplus T_{x}^{*} M$ corresponds exactly to the adjoint action of $\exp (Z)$. In particular, this adjoint action is an automorphism of the Lie algebra $\mathfrak{g}$ so the independence of the choice of the preferred connection follows.

In terms of triples, the filtration of $\mathcal{A}$ from 4.7 is simple to describe: $\mathcal{A}^{0}$ corresponds to triples of the form $(0, \Phi, \omega)$, while $\mathcal{A}^{1}$ corresponds to triples of the form $(0,0, \omega)$. From proposition 4.8 it is obvious that having one of these properties is independent of the choice of the preferred connection $\nabla$. But from the definition of the algebraic bracket it is obvious that $\mathcal{A}_{x}^{0}$ and $\mathcal{A}_{x}^{1}$ are subalgebras of $\mathcal{A}_{x}$, so we have a bundle of filtered Lie-algebras.

4.11. Next, we construct a tractor connection on $\mathcal{A}$. First observe that any preferred connection on $M$ gives rise to a connection on $\mathcal{A}$ (which is not a tractor connection) as follows: A preferred connection $\nabla$ gives a connection on $T M \oplus$ $\operatorname{End}_{0} T M \oplus T^{*} M$ which we can pull back, via the isomorphism of that bundle to $\mathcal{A}$ induced by $\nabla$, to get a connection on $\mathcal{A}$. We denote this connection also by $\nabla$. 
This just means that in the splitting into triples corresponding to $\nabla$ we have

$$
\nabla_{\eta}(\xi, \Phi, \omega)=\left(\nabla_{\eta} \xi, \nabla_{\eta} \Phi, \nabla_{\eta} \omega\right) .
$$

While this connection is not invariant (see below for the transformation law), it is compatible with the algebraic bracket on $\mathcal{A}$, i.e. $\nabla_{\eta}\{s, t\}=\left\{\nabla_{\eta} s, t\right\}+\left\{s, \nabla_{\eta} t\right\}$ for all section $s, t$ of $\mathcal{A}$. This is an obvious consequence of the componentwise definition of the algebraic bracket in 4.10 and the fact that $\nabla$ is compatible with the algebraic brackets on the factors, see 4.5. From here on, we use the convention that when computing in triples, we replace a triple which has only one nonzero entry by that entry. For example, we write $\Upsilon$ for $(0,0, \Upsilon),\{\Upsilon, \eta\}$ for $(0,\{\Upsilon, \eta\}, 0)$ and so on, if $\Upsilon$ is an element in or a section of $T^{*} M$ and $\eta$ is an element in or a section of $T M$ respectively. Note that this is compatible with the algebraic bracket by the componentwise definition.

Note that in this notation we can write the transformation laws for tractors and connections as

$$
(\widehat{\xi, \Phi, \omega})=(\xi, \Phi, \omega)+\{\Upsilon,(\xi, \Phi, \omega)\}+\frac{1}{2}\{\Upsilon,\{\Upsilon,(\xi, \Phi, \omega)\}\}
$$

and

$$
\hat{\nabla}_{\eta}(\xi, \Phi, \omega)=\nabla_{\eta}(\xi, \Phi, \omega)+\{\{\Upsilon, \eta\},(\xi, \Phi, \omega)\},
$$

respectively. Using this and the compatibility of $\nabla$ with the bracket, we now compute

$$
\left\{\Upsilon, \nabla_{\eta}(\xi, \Phi, \omega)\right\}=\nabla_{\eta}\{\Upsilon,(\xi, \Phi, \omega)\}-\left\{\nabla_{\eta} \Upsilon,(\xi, \Phi, \omega)\right\}
$$

and

$$
\frac{1}{2}\left\{\Upsilon,\left\{\Upsilon, \nabla_{\eta}(\xi, \Phi, \omega)\right\}\right\}=\frac{1}{2} \nabla_{\eta}\{\Upsilon,\{\Upsilon,(\xi, \Phi, \omega)\}\}-\left\{\nabla_{\eta} \Upsilon,\{\Upsilon,(\xi, \Phi, \omega)\}\right\}
$$

Using this, we see that

$$
\nabla_{\eta} \widehat{(\xi, \Phi, \omega)}=\nabla_{\eta}((\widehat{\xi, \Phi, \omega}))-\left\{\nabla_{\eta} \Upsilon,(\xi, \Phi, \omega)\right\}-\left\{\nabla_{\eta} \Upsilon,\{\Upsilon,(\xi, \Phi, \omega)\}\right\} .
$$

But since $\left\{\nabla_{\eta} \Upsilon,\{\Upsilon,\{\Upsilon,(\xi, \Phi, \omega)\}\}\right\}=0$, we may write this also as

$$
\nabla_{\eta} \widehat{(\xi, \Phi, \omega)}=\nabla_{\eta}((\widehat{\xi, \Phi, \omega}))-\left\{\nabla_{\eta} \Upsilon,(\widehat{\xi, \Phi, \omega})\right\}
$$

On the other hand, we have

$$
\hat{\nabla}_{\eta}((\widehat{\xi, \Phi, \omega}))=\nabla_{\eta}((\widehat{\xi, \Phi, \omega}))+\{\{\Upsilon, \eta\},(\widehat{\xi, \Phi, \omega})\}
$$

so we arrive at the transformation law

$$
\nabla_{\eta} \widehat{(\xi, \Phi, \omega)}-\hat{\nabla}_{\eta}((\widehat{\xi, \Phi, \omega}))=-\left\{\left(0,\{\Upsilon, \eta\}, \nabla_{\eta} \Upsilon\right),(\widehat{\xi, \Phi, \omega})\right\} .
$$

Theorem 4.12. The expression

$$
\nabla_{\eta}^{\mathcal{A}}(\xi, \Phi, \omega)=\nabla_{\eta}(\xi, \Phi, \omega)+\{(\eta, 0, \mathrm{P}(\eta)),(\xi, \Phi, \omega)\}
$$

in the splitting corresponding to $\nabla$ defines a tractor connection on $\mathcal{A}$ which is independent of the choice of the preferred connection $\nabla$.

Proof. Obviously, the expression defines a linear connection on $\mathcal{A}$. To see that the connection is independent of the choice of $\nabla$, we have to compute the transformation law for the second term. By the invariance of the bracket, this transformation is given by

$$
\{(\eta, \widehat{0, \mathrm{P}(\eta})),(\widehat{\xi, \Phi, \omega})\}=\left\{\left(\eta,\{\Upsilon, \eta\}, \mathrm{P}(\eta)+\frac{1}{2}\{\Upsilon,\{\Upsilon, \eta\}\}\right),(\widehat{\xi, \Phi, \omega})\right\} .
$$


On the other hand, we have

$$
(\eta, 0, \hat{\mathrm{P}}(\eta))=\left(\eta, 0, \mathrm{P}(\eta)-\nabla_{\eta} \Upsilon+\frac{1}{2}\{\Upsilon,\{\Upsilon, \eta\}\}\right) .
$$

Subtracting this from the above term, we see that the contribution of this part exactly cancels with the contribution from the last formula in section 4.11 so $\nabla^{\mathcal{A}}$ is independent of the choice of $\eta$.

To verify that $\nabla^{\mathcal{A}}$ is a tractor connection, we have to prove compatibility with the bracket and the non-degeneracy condition. Non-degeneracy follows immediately from the fact that $\nabla_{\eta}^{\mathcal{A}}(0,0, \omega)=\left(0,\{\eta, \omega\}, \nabla_{\eta} \omega\right)$. So the algebraic map $T M \rightarrow$ $\mathcal{A} / \mathcal{A}^{1}$ induced by $\omega \in \Omega^{1}(M)$ has values in $\operatorname{End}_{0} T M$ and is given by the algebraic bracket with $\omega$, so these maps (as we range over all $\omega$ ) separate points in $T M$; see 33, lemma 3.2]. Compatibility with the algebraic bracket for the first term in the definition of $\nabla^{\mathcal{A}}$ has been observed in 4.11 above, while for the second term it follows from the Jacobi identity. Hence $\nabla^{\mathcal{A}}$ is compatible with the algebraic bracket, so it is a $\mathfrak{g}$-connection by proposition 2.6 and thus a tractor connection.

4.13. The curvature of the tractor connection. We can now directly compute the curvature of the tractor connection $\nabla^{\mathcal{A}}$. First, by definition we have

$$
\nabla_{[\eta, \zeta]}^{\mathcal{A}}(\xi, \Phi, \omega)=\nabla_{[\eta, \zeta]}(\xi, \Phi, \omega)+\{([\eta, \zeta], 0, \mathrm{P}([\eta, \zeta])),(\xi, \Phi, \omega)\} .
$$

On the other hand we compute

$$
\nabla_{\eta}^{\mathcal{A}} \nabla_{\zeta}(\xi, \Phi, \omega)=\nabla_{\eta} \nabla_{\zeta}(\xi, \Phi, \omega)+\left\{(\eta, 0, \mathrm{P}(\eta)), \nabla_{\zeta}(\xi, \Phi, \omega)\right\}
$$

Finally, the compatibility of $\nabla^{\mathcal{A}}$ with the bracket implies

$$
\begin{aligned}
& \nabla_{\eta}^{\mathcal{A}}(\{(\zeta, 0, \mathrm{P}(\zeta)),(\xi, \Phi, \omega)\})=\left\{\nabla_{\eta}^{\mathcal{A}}(\zeta, 0, \mathrm{P}(\zeta)),(\xi, \Phi, \omega)\right\} \\
& \quad+\left\{(\zeta, 0, \mathrm{P}(\zeta)), \nabla_{\eta}(\xi, \Phi, \omega)\right\}+\{(\zeta, 0, \mathrm{P}(\zeta)),\{(\eta, 0, \mathrm{P}(\eta)),(\xi, \Phi, \omega)\}\}
\end{aligned}
$$

To get the curvature, we have to add the terms in (5) and (6) and then subtract the same terms with $\eta$ and $\zeta$ exchanged as well as the terms in (4). First note that the second term in (5) after alternation cancels with the second term in (6). Expanding the definition of $\nabla^{\mathcal{A}} \eta$ and using the Jacobi identity, we see that the remaining two terms in (6) add up to

$$
\left\{\nabla_{\eta}(\zeta, 0, \mathrm{P}(\zeta)),(\xi, \Phi, \omega)\right\}+\{(\eta, 0, \mathrm{P}(\eta)),\{(\zeta, 0, \mathrm{P}(\zeta)),(\xi, \Phi, \omega)\}\} .
$$

After alternation, the second of these terms just produces the bracket of

$$
\{(\eta, 0, \mathrm{P}(\eta)),(\zeta, 0, \mathrm{P}(\zeta))\}=(0,\{\eta, \mathrm{P}(\zeta)\}-\{\zeta, \mathrm{P}(\eta)\}, 0)
$$

with $(\xi, \Phi, \omega)$. The first term in (5) together with its alternation and subtracting the first term in (4) combine to give the curvature of $\nabla$ applied to $(\xi, \Phi, \omega)$, which can be written as $\{(0, R(\eta, \zeta), 0),(\xi, \Phi, \omega)\}$. By definition of the Weyl curvature (see 4.6) this combines with the term from above to yield $\{(0, W(\eta, \zeta), 0),(\xi, \Phi, \omega)\}$. The rest is then simply given by the bracket of

$$
\begin{aligned}
\nabla_{\eta}(\zeta, 0, \mathrm{P}(\zeta))- & \nabla_{\zeta}(\eta, 0, \mathrm{P}(\eta))-([\eta, \zeta], 0, \mathrm{P}([\eta, \zeta])) \\
& =\left(T(\eta, \zeta), 0, \nabla_{\eta}(\mathrm{P}(\zeta))-\nabla_{\zeta}(\mathrm{P}(\eta))-\mathrm{P}([\eta, \zeta])\right)
\end{aligned}
$$

with $(\xi, \Phi, \omega)$. Inserting $[\eta, \zeta]=\nabla_{\eta} \zeta-\nabla_{\zeta} \eta-T(\eta, \zeta)$, the last component in the right hand side can be written as $\left(\nabla_{\eta} \mathrm{P}\right)(\zeta)-\left(\nabla_{\zeta} \mathrm{P}\right)(\eta)+\mathrm{P}(T(\eta, \zeta))$, so we exactly 
recover the Cotton-York tensor of $\nabla$ from 4.6] Thus, the curvature $R^{\mathcal{A}}$ of $\nabla^{\mathcal{A}}$ is given by

$$
R^{\mathcal{A}}(\eta, \zeta)(t)=\{(T(\eta, \zeta), W(\eta, \zeta), C Y(\eta, \zeta)), t\} .
$$

Since by construction $T$ and $W$ are $\partial^{*}$-closed, while for $C Y$ this is trivially true, we have verified that $\nabla^{\mathcal{A}}$ is the normal tractor connection on $M$. Finally note that this shows that $\kappa(\eta, \zeta)$ is given by $(T(\eta, \zeta), W(\eta, \zeta), C Y(\eta, \zeta))$ in the splitting corresponding to $\nabla$. Since $\kappa(\xi, \eta)$ is a section of the adjoint tractor bundle by 2.9. proposition 4.8 implies the transformation law for the Cotton-York tensor claimed in 4.6 .

4.14. The fundamental $D$-operator on adjoint tractors and tensors. Having the normal tractor connection at hand, we can now easily compute the fundamental $D$-operators for a large class of bundles. Let us start with the fundamental $D$ on adjoint tractors themselves. From proposition 3.2(2) we know that for smooth sections $s, t \in \Gamma(\mathcal{A})$, we have $D_{s} t=\nabla_{\Pi(s)}^{\mathcal{A}} t-\{s, t\}$. If $\nabla$ is a preferred connection and in the splitting corresponding to $\nabla$ we have $s=(\xi, \Phi, \omega)$ and $t=(\eta, \Psi, \tau)$, then $\Pi(s)=\xi$ and the formula for $\nabla^{\mathcal{A}}$ from 4.12 immediately implies that in the splitting corresponding to $\nabla$, the section $D_{s} t$ is given by

$$
\begin{aligned}
& \left(\nabla_{\xi} \eta, \nabla_{\xi} \Psi, \nabla_{\xi} \tau\right)-\{(0, \Phi, \omega-\mathrm{P}(\xi)),(\eta, \Psi, \tau)\} \\
& =\left(\nabla_{\xi} \eta-\{\Phi, \eta\}, \nabla_{\xi} \Psi-\{\Phi, \Psi\}-\{\omega-\mathrm{P}(\xi), \eta\}, \nabla_{\xi} \tau-\{\Phi, \tau\}-\{\omega-\mathrm{P}(\xi), \Psi\}\right) .
\end{aligned}
$$

By proposition $3.1(2)$ we immediately see from this that the fundamental $D$ operators on vector fields and one-forms are given by $D_{s} \eta=\nabla_{\xi} \eta-\{\Phi, \eta\}$ and $D_{s} \tau=\nabla_{\xi} \tau-\{\Phi, \tau\}$, respectively. Let us introduce a final piece of notation. Any irreducible bundle $\mathcal{W}$ corresponds to a representation of $G_{0}$, so the corresponding infinitesimal representation induces a bundle map $\operatorname{End}_{0} T M \times \mathcal{W} \rightarrow \mathcal{W}$, which we denote by $\bullet$. Again, in the case of a tractor bundle this is compatible with our earlier notation. In this notation we can write $D_{s} \eta=\nabla_{\xi} \eta-\Phi \bullet \eta$ and $D_{s} \tau=\nabla_{\xi} \tau-\Phi \bullet \tau$. By naturality of the fundamental $D$-operators (see proposition [3.1(2) and (3)), this implies that $D_{s} t=\nabla_{\xi} t-\Phi \bullet t$ for any tensor field $t$ on $M$. Finally, for later use, observe that by its definition it is clear that for $\Phi \in \operatorname{End}_{0} T M$, $\Phi \bullet$ satisfies a Leibniz rule over the tensor product of induced bundles. For example if $\mathcal{W}$ and $\mathcal{W}^{\prime}$ are two irreducible bundles corresponding to two representations of $G_{0}$ and $s \otimes t \in \Gamma(\mathcal{W}) \otimes \Gamma\left(\mathcal{W}^{\prime}\right)$, then $\Phi \bullet(s \otimes t)=(\Phi \bullet s) \otimes t+s \otimes(\Phi \bullet t)$

To see how to proceed to compute iterations of fundamental $D$-operators, let us compute $D D t$ for any tensor field $t$ on $M$. Applying proposition 3.1(3) to the map $s_{2} \otimes D t \mapsto D_{s_{2}} t$ we immediately see that $D D t\left(s_{1}, s_{2}\right)=D_{s_{1}}\left(D_{s_{2}} t\right)-D_{D_{s_{1}} s_{2}} t$. If, in the splitting corresponding to a preferred connection $\nabla$, the sections $s_{i}$ of $\mathcal{A}$ are given by $\left(\xi_{i}, \Phi_{i}, \omega_{i}\right)$ for $i=1,2$, then we can read off $D_{s_{1}} s_{2}$ from above, so the second summand contributes

$$
-\nabla_{\nabla_{\xi_{1}} \xi_{2}} t+\nabla_{\left\{\Phi_{1}, \xi_{2}\right\}} t+\left(\nabla_{\xi_{1}} \Phi_{2}-\left\{\Phi_{1}, \Phi_{2}\right\}-\left\{\omega_{1}-\mathrm{P}\left(\xi_{1}\right), \xi_{2}\right\}\right) \bullet t .
$$

On the other hand, $D_{s_{1}}\left(\nabla_{\xi_{2}} t\right)=\nabla_{\xi_{1}} \nabla_{\xi_{2}} t-\Phi_{1} \bullet \nabla_{\xi_{2}} t$ and by naturality of the fundamental $D$ 's we get $D_{s_{1}}\left(\Phi_{2} \bullet t\right)=\left(D_{s_{1}} \Phi_{2}\right) \bullet t+\Phi_{2} \bullet\left(D_{s_{1}} t\right)$. Expanding the remaining $D$ 's and collecting terms, we see that $D D t\left(s_{1}, s_{2}\right)$ is given by

$$
\begin{aligned}
\nabla^{2} t\left(\xi_{1}, \xi_{2}\right) & -\Phi_{1} \bullet \nabla_{\xi_{2}} t-\Phi_{2} \bullet \nabla_{\xi_{1}} t+\nabla_{\left\{\Phi_{1}, \xi_{2}\right\}} t \\
& +\Phi_{2} \bullet \Phi_{1} \bullet t-\left\{\omega_{1}, \xi_{2}\right\} \bullet t+\left\{\mathrm{P}\left(\xi_{1}\right), \xi_{2}\right\} \bullet t .
\end{aligned}
$$


4.15. Natural line bundles and weighted tensor fields. In the case of irreducible parabolic geometries, natural line bundles are also easy to understand. First observe that the adjoint action induces a representation $\mathrm{Ad}_{-}: G_{0} \rightarrow G L\left(\mathfrak{g}_{-1}\right)$. Now for a real number $w$ we define the bundle $\mathcal{E}[w]$ to be the line bundle associated to $\mathcal{G}_{0}$ corresponding to the representation $g \mapsto\left|\operatorname{det}\left(\operatorname{Ad}_{-}(g)\right)\right|^{-w / n}$ where $n=\operatorname{dim}\left(\mathfrak{g}_{-1}\right)$. Otherwise put, $\mathcal{E}[w]$ is the bundle of $-w / n$-densities on $M$. The choice of the normalization is motivated as follows: The corresponding infinitesimal representation is given by $-w / n$ times the trace of the restriction of ad to $\mathfrak{g}_{-1}$. Since by definition the grading element acts as the negative of the identity on $\mathfrak{g}_{-1}$, the number $w$ is exactly the value of the infinitesimal representation on the grading element. Since the center of $\mathfrak{g}_{0}$ is 1 -dimensional in the $|1|$-graded case, any natural line bundle is locally isomorphic to some $\mathcal{E}[w]$, so it suffices to compute the fundamental $D$ 's for these bundles.

By construction, the highest exterior power $\Lambda^{\max } T^{*} M=\Lambda^{n} T^{*} M$ of $T^{*} M$ is locally isomorphic to $\mathcal{E}[n]$. Since this is a tensor bundle, we know from 4.14 above, that for sections $s \in \Gamma(\mathcal{A})$ and $\sigma \in \Gamma\left(\Lambda^{n} T^{*} M\right)$, and a preferred connection $\nabla$, we have $D_{s} \sigma=\nabla_{\xi} \sigma-\Phi \bullet \sigma$, where $s=(\xi, \Phi, \omega)$ in the splitting corresponding to $\nabla$. By the definition of the fundamental $D$-operators, the same formula holds for $\sigma \in \Gamma(\mathcal{E}[n])$. Of course by proposition 3.2 (1) this formula also holds for the trivial line bundle $\mathcal{E}[0]$ with the space of smooth sections $C^{\infty}(M, \mathbb{R})$. Now let $0 \neq \alpha \in \mathbb{R}$ and suppose $\mu$ is any section of $\mathcal{E}\left[\frac{n}{\alpha}\right]$. From the previous but one result we have $D_{s} \mu^{\alpha}=\nabla_{\xi} \mu^{\alpha}-\Phi \bullet \mu^{\alpha}$. By definition of the bundle $\mathcal{E}[w]$, for $\sigma \in \Gamma(\mathcal{E}[w])$, we have $\Phi \bullet \sigma=-\frac{w}{n} \operatorname{tr}(\Phi) \sigma$. In particular $\Phi \bullet \mu^{\alpha}=\alpha \mu^{\alpha-1} \Phi \bullet \mu$. Of course $\nabla_{\xi} \mu^{\alpha}=\alpha \mu^{\alpha-1} \nabla_{\xi} \mu$, and by proposition 3.5 (3), we have $D_{s} \mu^{\alpha}=\alpha \mu^{\alpha-1} D_{s} \mu$. Thus we may conclude that the formula $D_{s} \mu=\nabla_{\xi} \mu-\Phi \bullet \mu$ holds for sections of any line bundle.

Proposition. (1) Let $t$ be a section of the tensor product of a tensor bundle with a natural line bundle. Then

$$
D_{s} t=\nabla_{\xi} t-\Phi \bullet t
$$

where $s \in \Gamma(\mathcal{A})$ is given by $(\xi, \Phi, \omega)$ in the splitting corresponding to $\nabla$. Similarly the formula for DDt derived in 4.14 continues to hold for weighted tensor fields.

(2) If $\sigma$ is a section of the bundle $\mathcal{E}[w]$ for some $w \in \mathbb{R}$, then

$$
D_{s} \sigma=\nabla_{\xi} \sigma+\frac{w}{n} \operatorname{tr}(\Phi) \sigma
$$

where, again, $s=(\xi, \Phi, \omega)$ in the splitting corresponding to $\nabla$.

(3) Let $\sigma$ be a non-vanishing section of the bundle $\mathcal{E}[w]$ for $0 \neq w \in \mathbb{R}$ and let $E \in \mathfrak{g}_{0}$ be the grading element. Then $B(E, E) \neq 0$ and for a preferred connection $\nabla$ the tractor $A^{\sigma}$ from proposition $3.5(1)$ is given in the splitting corresponding to $\nabla$ by $\frac{w}{B(E, E)}\left(0, \mathrm{id}, \sigma^{-1} \nabla \sigma\right)$.

Proof. The first of these follows immediately from the results for tensors and line bundles since $D$ satisfies the Leibniz rule for tensor products of induced bundles.

For (2) simply observe that by definition of $\mathcal{E}[w]$, we have $\Phi \bullet \sigma=-\frac{w}{n} \operatorname{tr}(\Phi) \sigma$ for all sections $\Phi$ of $\operatorname{End}_{0} T M$.

Finally we deal with (3). Let $C$ be the Killing form on $\mathfrak{g}$. Since the grading element $E$ acts by multiplication by $j$ on each $\mathfrak{g}_{j}$, the definition of the Killing form implies $C(E, E)=2 n$, where $n$ denotes the dimension of $\mathfrak{g}_{-1}$. Since $B$ is a nonzero multiple of the Killing form, we also have $B(E, E) \neq 0$. 
Now for any smooth section $s$ of $\mathcal{A}$, we know from above that we have $D_{s} \sigma=$ $\nabla_{\xi} \sigma-\Phi \bullet \sigma=B\left(A^{\sigma}, s\right) \sigma$, where $s=(\xi, \Phi, \omega)$ in the splitting corresponding to $\nabla$. Next, consider the one-form $\Upsilon:=\sigma^{-1} \nabla \sigma$. If $\hat{\nabla}$ denotes the connection obtained by deforming $\nabla$ corresponding to $-\Upsilon$, then by construction $\hat{\nabla} \sigma=0$, and clearly $\hat{\nabla}$ is the unique preferred connection which has this property. Hence we see that $D_{s} \sigma=-\Psi \bullet \sigma=B\left(A^{\sigma}, s\right) \sigma$, where $s=(\xi, \Psi, \tau)$ in the splitting corresponding to $\hat{\nabla}$. Recall, once again, that $\Psi \bullet \sigma=-\frac{w}{n} \operatorname{tr}(\Psi) \sigma$ for all sections $\Psi$ of $\operatorname{End}_{0} T M$ and $\sigma \in$ $\Gamma(\mathcal{E}[w])$. So in the splitting corresponding to $\hat{\nabla}$, we must have $B\left(A^{\sigma},(\xi, \Psi, \tau)\right)=$ $\frac{w}{n} \operatorname{tr}(\Psi)$. On the other hand, for $A \in \mathfrak{g}_{0}$, by definition of the Killing form, we get $\stackrel{C}{C}(E, A)=\operatorname{tr}\left(\left.\operatorname{ad}(-A)\right|_{\mathfrak{g}_{-1}}\right)+\operatorname{tr}\left(\left.\operatorname{ad}(A)\right|_{\mathfrak{g}_{1}}\right)$, and from the definition of the adjoint action one easily verifies that this equals $-2 \operatorname{tr}\left(\left.\operatorname{ad}(A)\right|_{\mathfrak{g}_{-1}}\right)$. Since $B$ is a multiple of the Killing form and $C(E, E)=2 n$, we get $B(E, A)=\frac{B(E, E)}{2 n} C(E, A)$. Since the constant function $E$ corresponds to - id $\in \Gamma\left(\operatorname{End}_{0} T M\right)$, we see that in the splitting corresponding to $\hat{\nabla}$, the adjoint tractor $\left(0, \frac{w}{B(E, E)}\right.$ id, 0$)$ has the property that its pairing under $B$ with some $\Psi$ gives exactly $\frac{w}{n} \operatorname{tr}(\Psi)$, so by the uniqueness part of proposition [3.5(1), it coincides with $A^{\sigma}$. Passing back to the splitting corresponding to the connection $\nabla$, proposition 4.8 implies that $A^{\sigma}$ is given by $\left(0, \frac{w}{B(E, E)}\right.$ id, $\left\{\Upsilon, \frac{w}{B(E, E)}\right.$ id $\left.\}\right)$. Since the bracket $T^{*} M \otimes \operatorname{End}_{0} T M \rightarrow T^{*} M$ is given exactly by the evaluation of endomorphisms, the last component equals $\frac{w}{B(E, E)} \Upsilon=$ $\frac{w}{B(E, E)} \sigma^{-1} \nabla \sigma$, and the result follows.

Remark. (1) In the proof of the above proposition we have seen that for any nonvanishing section $\sigma$ (or equivalently any trivialization) of one of the bundles $\mathcal{E}[w]$ with $w \neq 0$ there is a unique preferred connection $\nabla$ such that $\nabla \sigma=0$. The connections obtained in that way form a proper subclass of all preferred connections (since they are affine over smooth functions). In the case of conformal structures these are exactly the Levi-Civita connections of metrics in the conformal class (as opposed to Weyl-structures, which are the general preferred connections). Note that it follows easily from the definition of $A^{\sigma}$ in proposition 3.5 (1) that the adjoint adjoint tractor $\frac{B(E, E)}{w} A^{\sigma}$ is independent of the scale of the form $B$ and the power of $\sigma$. That is if $\alpha \neq 0$, then $\frac{B(E, E)}{\alpha w} A^{\sigma^{\alpha}}=\frac{B(E, E)}{w} A^{\sigma}$. Moreover, in the proof we saw that in the splitting corresponding to the preferred connection $\nabla$ with $\nabla \sigma=0$, the adjoint tractor $-\frac{B(E, E)}{w} A^{\sigma}$ is simply given by $(0,-\mathrm{id}, 0)$. In particular, this implies that the isomorphism $\mathcal{A} \cong T M \oplus \operatorname{End}_{0} T M \oplus T^{*} M$ induced by $\nabla$ is exactly the splitting of $\mathcal{A}$ into the eigenspaces corresponding to the eigenvalues $-1,0,1$ of the operator $-\frac{B(E, E)}{w}\left\{A^{\sigma},\right\}$.

(2) Suppose that we write $\tilde{w}$ for the representation inducing the line bundle $\mathcal{E}[w]$. Let us also write $\tilde{w}$ for the corresponding representation of the Lie algebra $\mathfrak{g}_{0}$. Then it follows immediately from the previous observation that $w=\tilde{w}(E)$, where $E$ is the grading element. This gives an alternative understanding of the density weight for irreducible parabolic structures. Another weight is commonly used for these bundles. In the complexification of $\mathfrak{g}$ the parabolic subalgebra (the complexification of $\mathfrak{p}$ ) corresponds to omitting the weight spaces corresponding to all negative roots which are linear combinations involving a certain simple root $\alpha_{\times}$(see [25] for this and other notation used here). In fact this is the simple root such that $\alpha(E)=1$. (We are assuming here that the Cartan subalgebra is consistent with the given grading.) Corresponding to each simple root $\alpha$ there is 
an element, usually denoted $h_{\alpha}$, of a standard basis of the Cartan subalgebra. The representation $\tilde{w}$ on $\mathfrak{g}$ is determined by $\tilde{w}\left(h_{\alpha_{\times}}\right)$and this number is often used as a characterizing weight for the representation. For example this is the convention used in [2, 21]. The difference between $\tilde{w}\left(h_{\alpha_{x}}\right)$ and $\tilde{w}(E)$ depends on the structure. More precisely, if one takes the basis $\left\{h_{\alpha}\right\}$ of the Cartan algebra, where $\alpha$ ranges over the set of simple roots, then $E$ is the element of the dual basis (with respect to the Killing form) corresponding to $h_{\alpha_{\times}}$. So the difference is exactly given by the coefficient of $h_{\alpha_{\times}}$in the expression of $E$ in the basis $\left\{h_{\alpha}\right\}$. Since it is well known that this coefficient can be read off the inverse of the Cartan matrix, this poses no problem. For example these numbers agree on conformal geometries, while on projective geometries $\tilde{w}\left(h_{\alpha_{\times}}\right)$is $\frac{n+1}{n} \tilde{w}(E)$.

\section{REFERENCES}

[1] T.N. Bailey and M.G. Eastwood, Complex paraconformal manifolds: their differential geometry and twistor theory, Forum Math. 3 (1991), 61-103. MR 92a:32038

[2] T.N. Bailey, M.G. Eastwood, A.R. Gover, Thomas's structure bundle for conformal, projective and related structures, Rocky Mountain J. 24 (1994), 1191-1217. MR 96e:53016

[3] T.N. Bailey, M.G. Eastwood and C. R. Graham, Invariant theory for conformal and CR geometry. Ann. of Math. 139 (1994), 491-552. MR 95h:53016

[4] R. J. Baston, Almost Hermitian symmetric manifolds, I: Local twistor theory, II: Differential Invariants, Duke Math. J. 63 (1991), 81-111, 113-138. MR 93d:53064

[5] T. Branson, A.R. Gover, Conformally Invariant Non-Local Operators, to appear in Pacific J. Math.

[6] A. Čap, H. Schichl, Parabolic Geometries and Canonical Cartan Connections, Hokkaido Math. J. 29 No.3 (2000), 453-505. CMP 2001:04

[7] A. Čap, J. Slovák, On local flatness of manifolds with AHS-structures in Proc. of the Winter School Geometry and Physics, Srni 1995 Supp ai Rend. Circ. Mat. Palermo 43 (1996), 95-101. MR 98j:53059

[8] A. Čap, J. Slovák, V. Souček, Invariant operators on manifolds with almost Hermitian symmetric structures, I. invariant differentiation, Acta Math. Univ. Commenianae, 66 No. 1 (1997), 33-69, electronically available at http://www.emis.de; MR 98m:53036

II. normal Cartan connections, Acta Math. Univ. Commenianae, 66 No. 2 (1997), 203-220, electronically available at http://www.emis.de; MR 2000a:53045 III. Standard operators, Diff. Geom. Appl. 12 No. 1 (2000) 51-84. MR 2001h:53038

[9] A. Čap, J. Slovák, V. Souček, Bernstein-Gelfand-Gelfand Sequences to appear in Ann. of Math., extended version electronically available as Preprint ESI 722 at http://www.esi.ac.at

[10] E. Cartan, Les espaces à connexion conforme, Ann. Soc. Pol. Math. 2 (1923), 171-202.

[11] K. Dighton, An introduction to the theory of local twistors, Int. J. Theor. Phys. 11 (1974), 31-43. MR 54:9475

[12] M.G. Eastwood, Notes on Conformal Differential Geometry, Supp. Rend. Circ. Matem. Palermo 43 (1996), 57-76. MR 98g:53021

[13] M.G. Eastwood and J.W. Rice, Conformally invariant differential operators on Minkowski space and their curved analogues, Commun. Math. Phys. 109 (1987), 207-228. Erratum:144 (1992), 213. MR 89d:22012 MR 92k:22026

[14] C. Fefferman, Monge-Ampère equations, the Bergman kernel and geometry of pseudoconvex domains, Ann. of Math. 103 (1976), 395-416; Erratum 104 (1976), 393-394. MR 53:11097a MR 53:11097b

[15] C. Fefferman and C.R. Graham, Conformal invariants, in Élie Cartan et les Mathématiques d'Adjourd'hui, (Astérisque, hors serie), (1985), 95-116. MR 87g:53060

[16] A.B. Goncharov, Generalized conformal structures on manifolds, Selecta Math. Soviet. 6 (1987), 308-340. MR 89e:53050

[17] A.R. Gover, Invariants and Calculus for Projective Geometries, Math. Ann. 306 (1996) 513-538. MR 97k:53019

[18] A.R. Gover, Invariants and calculus for conformal geometry, to appear in Adv. Math. 
[19] A.R. Gover, Aspects of parabolic invariant theory, in Proc. of the Winter School Geometry and Physics, Srni 1998 Supp. Rend. Circ. Matem. Palermo, Ser. II. Suppl. 59 (1999), 25-47. MR 2001a:58047

[20] A.R. Gover, K. Hirachi, In progress.

[21] A.R. Gover, J. Slovák, Invariant Local Twistor Calculus for Quaternionic Structures and Related Geometries, J. Geom. Phys. 32 (1999) 14-56. MR 2001b:53051

[22] A.R. Gover, C.R. Graham, CR calculus and invariant powers of the sub-Laplacian, In progress.

[23] Graham C.R.: Invariant theory of parabolic geometries in Komatsu, Sakane, (eds.) Complex Geometry. Proceedings Osaka 1990 (Marcel Dekker Lecture Notes in Pure and Applied Mathematics) New York, Basel, Hong Kong: Marcel Dekker, 1993. MR 94a:32013

[24] K. Hirachi, Construction of boundary invariants and the logarithmic singularity of the Bergman kernel, Ann. of Math. 151 no. 1 (2000), 151-191. MR 2001f:32003

[25] J.E. Humphreys, Introduction to Lie algebras and representation theory, Springer-Verlag, New York (1972). MR 48:2197

[26] B. Kostant, Lie algebra cohomology and the generalized Borel-Weil theorem, Ann. of Math. 74 no. 2 (1961), 329-387. MR 26:265

[27] T. Morimoto, Geometric structures on filtered manifolds, Hokkaido Math. J. 22 (1993), 263-347. MR 94m:58243

[28] T. Ochiai, Geometry associated with semisimple flat homogeneous spaces, Trans. Amer. Math. Soc. 152 (1970), 159-193. MR 44:2160

[29] J. Slovák, Conformal differential geometry lecture notes, University of Vienna, 1992, electronically available at http://www.math.muni.cz/ slovak.

[30] N. Tanaka, On the equivalence problem associated with a certain class of homogeneous spaces, J. Math. Soc. Japan 17 (1965), 103-139. MR 32:6358

[31] N. Tanaka, On the equivalence problem associated with simple graded Lie algebras, Hokkaido Math. J. 8 (1979), 23-84. MR 80h:53034

[32] T.Y. Thomas, On conformal geometry, Proc. N.A.S. 12 (1926), 352-359; Conformal tensors, Proc. N.A.S. 18 (1931), 103-189.

[33] K. Yamaguchi, Differential Systems Associated with Simple Graded Lie Algebras, Advanced Studies in Pure Math. 22 (1993), 413-494. MR 95g:58263

Institut für Mathematik, Universität Wien, Strudlhofgasse 4, A-1090 Wien, Austria and International Erwin Schrödinger Institute for Mathematical Physics, Boltzmanngasse 9, A-1090 Wien, Austria

E-mail address: Andreas.Cap@esi.ac.at

Department of Mathematics, The University of Auckland, Private Bag 92019, AuckLAND, NEW ZEALAND

E-mail address: gover@math.auckland.ac.nz 